\title{
FEM/SEA Hybrid Method for Predicting Mid and High Frequency Structure-Borne Transmission
}

\author{
B. Troclet ${ }^{*}, 1$, B. Hiverniau ${ }^{2}$, M.N. Ichchou ${ }^{2}$, L. Jezequel ${ }^{2}$, K. Kayvantash ${ }^{4}$, T. Bekkour ${ }^{3}$, \\ J.B. Mouillet ${ }^{3}$ and A. Gallet ${ }^{3}$ \\ ${ }^{I}$ EADS ASTRIUM ST, 66, Route de Verneuil, BP 300278133 Les Mureaux Cedex, France \\ ${ }^{2}$ Ecole Centrale de Lyon, 36, avenue Guy de Collongue 69134 Ecully Cedex, France \\ ${ }^{3}$ Altair Development France, 2, rue de la Renaissance, 92184 Antony, France \\ ${ }^{4}$ Cranfield University, Cranfield, Bedfordshire MK43 OAL, UK
}

\begin{abstract}
Launch Vehicles are subject, at lift-off and during flight ascent, to acoustic and aeroacoustic loads, which are random in nature. Because electronic units are very sensitive to mid and high frequency loads, it is important to numerically predict and specify the vibration levels to be applied to units for qualification test. The general objective of the activity presented in this paper is to develop a methodology to predict mid and high frequency structure-borne transmissions in launch vehicles. As the loads of interest are random, it has been chosen to investigate energy-based modeling approaches, combined with the Finite Element Method. For energy-based modeling, the structure is divided into subsystems. For high frequency predictions, the purely numerical Power Injection Method, derived from Statistical Energy Analysis, is used to estimate the Coupling Loss Factor between structural subsystems. For the mid frequency predictions, an approach close to Statistical Energy Analysis, called Statistical Energy Analysis-Like (SEA-like), is investigated. In this approach, a relation between total energies of subsystems and input powers is established, by identifying a matrix composed of Energy Influence Coefficients. The objective of the study is to establish the methodology to compute with accuracy, using the Finite Element Method, Coupling Loss Factors and Energy Influence Coefficient. It is shown that the excitation of subsystems by 'Rain on the Roof' loads defined by the 'Influence Circle' and the Optimal Latin Hypercube methods provide accurate coupling data. A validation of the methodology on academic and industrial cases is presented.
\end{abstract}

\section{INTRODUCTION}

Launch vehicles are subject to several types of broadband loads such as acoustic loads due to rocket engines, at lift-off, and aeroacoustic loads due to the external aerodynamic environment during flight ascent. Those excitations are random. The induced random acoustic and vibration environment may create physical discomfort or damage to the structures and electronic equipment. Consequently, it is very important to use reliable and predictive tools to estimate the response of launch vehicle structures to those loads. The specification of random vibration qualification levels to be applied to equipment during qualification tests before flights is an activity of great importance, given the severity of the in-flight environment.

The frequency band of interest ranges from $20 \mathrm{~Hz}$ up to $2000 \mathrm{~Hz}$. Consequently, several vibroacoustic methods have to be used in order to cover the full domain. For the low frequency regime, a mode by mode analysis is for example used [1], using the Finite Element Method, the fluid being represented by Green's function. In the high frequency domain, where high modal overlap occurs, SEA [2, 3], is

*Address correspondence to this author at the EADS ASTRIUM ST, 66, Route de Verneuil, BP 300278133 Les Mureaux Cedex, France;

E-mail: bernard.troclet@astrium.eads.net better suited [4]. A predicting gap exists in the mid frequency domain. Moreover, structure-borne transmissions are not well predicted. To bridge this mid frequency gap, a SEA-Like method [5], is proposed.

This paper focuses on structure-borne transmissions in the mid and high frequency domains for structural configurations subject to normal excitations, such as pressure fields. Very few closed formulations are available to estimate structure-borne transmissions between coupled structures encountered in industrial real life, except, for example, structure-borne transmissions between perpendicularly coupled homogeneous plates [6].

In this paper, we propose to perform this estimate using a purely numerical PIM method in the high frequency range [7-9], and a SEA-Like method in the mid-frequency range. The PIM method is an experimental method to estimate CLF of SEA by exciting the structure. This method has been investigated, described and justified in the literature [7-9]. But, as described in paragraph III, we intend to apply it to purely numerical models, as the real structure is not always available for experimental measurements.

In the mid frequency range, hybrid FEM/SEA approaches have already been investigated, using for instance classical modal analysis $[4,10]$, the influence of modal overlap has been shown and several cases where the 
indirect CLF had to be taken into account were pointed out. To solve those problems, we introduce in paragraph IV, the SEA-Like methodology to compute the Energy Influence Coefficients (EIC) between the structures.

After a general presentation of the classical SEA analysis, the methodology combining the Finite Element Method and energetic method, such as SEA for high frequency predictions and SEA-Like for mid frequency predictions, is presented in this paper, and validated on academic and industrial cases. The choice of SEA-Like method for predicting mid frequency responses is discussed in section 4. FEM is used to provide key parameters for SEA and SEA computations. As in the experimental PIM, the FEM model has to be correctly excited by forces to determine accurate CLF for SEA on one hand, and EIC for SEA-Like on the other hand. In our approach the FEM model is excited by a so-called 'Rain on The Roof' force field. However, the way of applying the forces is not standardized and we have adopted the method described in [7]. In this paper, it is stated that a number of three point loads are sufficient to obtain accurate coupling data whereas it is of course not sufficient to excite all modes of the structures in the mid frequency domain.

The key and innovative points of our method described in this paper are:

- $\quad$ The methodology proposed for exciting the FEM model to obtain accurate CLF for SEA and EIC for SEA-Like, based on the 'Influence Circle' method and on OLH method, in order to have a rational basis to estimate EIC and CLF. The 'Influence Circle' is presented later. The way of exciting the structure is a problem of great importance, because all modes in the frequency bands of concern have to be excited simultaneously in order to obtain accurate results.

- $\quad$ The computation in the time domain using an explicit code and its use in the context of energy methods. Indeed, explicit codes allow easy computations in time domaine, and provide mid \& high frequencies results (via adequate post-processing) with reasonable computation times for industrial structural configurations.

- $\quad$ The application of the methodology presented to real industrial case.

\section{PRESENTATION OF THE SEA METHOD}

Energy based methods are often applied to the characterization of the vibrating behaviour of structures in the high frequency domain. The most common approach is the SEA, Lyon [2, 3]. The method consists of decomposing a complete system into subsystems in order to estimate their total mean energy, averaged in time and space. This method is based on the energy equilibrium equation: the sum of the dissipated power in a given subsystem and the power exchanged with the other coupled subsystems is equal to the power supplied by the external loads to this subsystem. An important issue is the definition of a proper SEA model.
From the point of view adopted in this paper, a proper SEA model must satisfy the following four conditions:

The first condition is that the connected structural subsystems must have similar dynamic properties. The second condition is that the modal overlap criterion must be satisfied. This criterion for subsystem $i$ can be written as:

$n_{i} \eta_{i i} \omega \geq 1$

The third condition is that no indirect coupling occurs between structural subsystems. This condition leads to the absence of coupling between not directly coupled subsystems.

The fourth condition is that "weak coupling" condition has to be satisfied. The weak coupling hypothesis is the subject of large discussion in the SEA literature. However, we share the view of B. Mace [5], in that two coupled subsystems are in weak coupling condition, if the transmission, in the frequency band of concern, they involve local modes, and, as a consequence, do not involve global modes of the coupled subsystems.

The power flow between subsystems can be written as a relation between DLF, CLF and the modal densities of the subsystems. In the case of $\mathrm{n}$ coupled subsystems, a relation between the input powers and the total energy of the subsystems can be written in a matrix form, as follows:

$$
\left[\begin{array}{cccc}
\eta_{1 t o t} & -\eta_{12} & \cdots & -\eta_{1 n} \\
-\eta_{21} & \eta_{2 t o t} & \cdots & -\eta_{2 n} \\
\vdots & \vdots & \cdots & \vdots \\
-\eta_{n 1} & -\eta_{n 2} & \cdots & \eta_{n t o t}
\end{array}\right]\left[\begin{array}{c}
\frac{E_{1}}{n_{1}} \\
\frac{E_{2}}{n_{2}} \\
\vdots \\
\frac{E_{n}}{n_{n}}
\end{array}\right]=\left[\begin{array}{c}
\frac{P_{1, i n j}}{n_{1} \omega} \\
\frac{P_{2, i n j}}{n_{2} \omega} \\
\vdots \\
\frac{P_{n, i n j}}{n_{n} \omega}
\end{array}\right]
$$

The input powers $P_{i, i n j}$ are assumed to be known and characterized. Power inputs due to aero-acoustic loads are difficult to identify because the broadband pressure field has to be characterized with accuracy [4]. The characterization of the power inputs is out of the scope of this paper. If the modal densities of the subsystems, the DLF and the CLF, are known, the total energies of subsystems can be determined. The Dissipation Loss Factors are experimentally identified in most cases or extracted from a database. The modal densities of subsystems and the CLF per frequency bands must be estimated for solving the SEA matrix system (2). For estimating CLF between simply connected structures, the vibrational field in structures is represented by incoherent plane waves. The high frequency CLF are then obtained by computing the transmission coefficient between subsystems, averaged over all angles of incident plane waves [6]. However, this methodology does not provide satisfactory results if the connections are complex. An alternative method has, consequently, to be investigated. The methods of interest in this paper are the numerical PIM and the SEALike methods, for the high frequency and mid frequency domain respectively. 


\section{THE PIM METHOD}

For more complex structures, the CLF and DLF are often determined using an experimental approach such as the Power Injection Method (PIM). This approach has been largely investigated in the literature [7,9]. Once the vibrating total averaged energies and the power inputs are measured, an inverse energetic approach by writing SEA equations and inverting the SEA matrix of total energies can be applied. But a prototype of the structural configuration of interest is not always available to carry out such an experimental analysis. Consequently, the idea is to replace the experimental PIM by a numerical PIM, by combining FEM and SEA. This approach is called in this paper the hybrid FEM/SEA approach. The hybrid FEM/SEA approach is based on experimental PIM and consists of estimating the energies and the power injected by a FEM.

Let us consider a structural configuration composed of $n$ subsystems, of which a Finite Element Model has been realized. The conditions mentioned in paragraph II, in order to be a proper SEA model, are assumed to be satisfied. By using the reciprocity relation:

$\omega \eta_{i j} n_{i}=\omega \eta_{j i} n_{j}$

The matrix system defined in Eq. (2) can be rewritten:

$\omega *\left[\begin{array}{ccc}\eta_{1 t o t} & -\eta_{j 1} & -\eta_{n 1} \\ -\eta_{i j} & \eta_{j t o t} & -\eta_{n j} \\ -\eta_{1 n} & \ldots & \eta_{n t o t}\end{array}\right] *\left[\begin{array}{c}E_{1} \\ E_{j} \\ E_{n}\end{array}\right]=\left[\begin{array}{c}P_{1} \\ P_{j} \\ P_{n}\end{array}\right]$

The numerical PIM consists in numerically exciting the subsystems one after each others using predefined forces. The total energies of the subsystems, the time and space averaged power injected are estimated by forced response analysis using a FEM.

First, only subsystem 1 is excited. The injected power is denoted $P_{1}$. By normalizing the total energies by the injected power, the matrix system defined in Eq. (4) becomes:

$\omega *\left[\begin{array}{ccc}\eta_{1 t o t} & -\eta_{j 1} & -\eta_{n 1} \\ -\eta_{i j} & \eta_{j t o t} & -\eta_{n j} \\ -\eta_{1 n} & \ldots & \eta_{n t o t}\end{array}\right] *\left[\begin{array}{c}\left\langle E_{11}\right\rangle \\ \left\langle E_{j 1}\right\rangle \\ \left\langle E_{n 1}\right\rangle\end{array}\right]=\left[\begin{array}{l}1 \\ 0 \\ 0\end{array}\right]$

where:

$\left\langle E_{i 1}\right\rangle=\frac{E_{i 1}}{P_{1}}$

The $n-1$ other subsystems are then excited one by one and the following matrix system is obtained:

$\left[\begin{array}{cccc}\sum \eta_{1 i} & -\eta_{21} & \ldots & -\eta_{n 1} \\ -\eta_{12} & \sum \eta_{2 i} & \ldots & \ldots \\ \ldots & \ldots & \sum \eta_{i i} & \ldots \\ -\eta_{1 n} & \ldots & \ldots & \sum \eta_{n i}\end{array}\right] *\left[\begin{array}{cccc}\left\langle E_{11}\right\rangle & \left\langle E_{12}\right\rangle & \ldots & \left\langle E_{1 n}\right\rangle \\ \left\langle E_{21}\right\rangle & \left\langle E_{22}\right\rangle & \ldots & \ldots \\ \ldots \ldots & \ldots & \left\langle E_{k k}\right\rangle & \ldots \\ \left\langle E_{n 1}\right\rangle & \ldots & \ldots & \left\langle E_{m n}\right.\end{array}\right]=\frac{1}{\omega} *\left[\begin{array}{cccc}1 & 0 & \ldots & 0 \\ 0 & 1 & \ldots & \ldots \\ \ldots \ldots & \ldots & \ldots & \ldots \\ 0 & \ldots & \ldots & 1\end{array}\right]$

With:

$\left\langle E_{i j}\right\rangle=\frac{E_{i j}}{P_{J}}$
The coupling loss factors can be obtained by inversing the matrix $\left[\left\langle E_{i j}\right\rangle\right]$ :

$\left[\begin{array}{cccc}\sum \eta_{1 i} & -\eta_{21} & \ldots & -\eta_{n 1} \\ -\eta_{12} & \sum \eta_{2 i} & \ldots & \ldots \\ \ldots & \ldots & \sum \eta_{k i} & \ldots \\ -\eta_{1 n} & \ldots & \ldots & \sum \eta_{n i}\end{array}\right]=\frac{1}{\omega} *\left[\begin{array}{cccc}\left\langle E_{11}\right\rangle & \left\langle E_{12}\right\rangle & \ldots & \left\langle E_{1 n}\right\rangle \\ \left\langle E_{21}\right\rangle & \left\langle E_{22}\right\rangle & \ldots & \ldots \\ \ldots & \ldots & \left\langle E_{k k}\right\rangle & \ldots \\ \left\langle E_{n 1}\right\rangle & \ldots & \ldots & \left\langle E_{n n}\right\rangle\end{array}\right]^{-1}$

The matrix system defined in Eq. (8) can be written in a simplified form as follows:

$[\eta]=\frac{1}{\omega} *[\langle E\rangle]^{-1}$

The inversion of the total energies matrix can involve numerical errors due to ill-conditioning of the matrix and may lead to non-physical results. According to the main hypothesis of a proper SEA model, no indirect coupling exists. As a consequence, the CLF between two connected subsystems can be determined by extracting the two subsystems from the complete model and by exciting these two subsystems one by one. The application of the Lalor development [11] consists in solving two separate systems for the DLF and the CLF. The CLF can be written as:

$\eta_{i j}=\frac{1}{\omega}\left(\frac{\left\langle E^{n}{ }_{i i}\right\rangle}{\left\langle E^{n}{ }_{j i}\right\rangle}-\frac{\left\langle E^{n}{ }_{i j}\right\rangle}{\left\langle E^{n}{ }_{j j}\right\rangle}\right)^{-1} \frac{1}{\left\langle E^{n}{ }_{j j}\right\rangle}$

In the case of weak coupling, ie:

$\left\langle E_{i j}{ }^{n}\right\rangle \prec \prec\left\langle E_{j j}{ }^{n}\right\rangle$

Eq. (10) can be simplified as follows:

$\eta_{i j}=\frac{1}{\omega} \frac{\left\langle E_{j i}{ }^{n}\right\rangle_{\Delta \omega, s}}{\left\langle E_{i i}{ }^{n}\right\rangle_{\Delta \omega, s} \cdot\left\langle E_{j j}{ }^{n}\right\rangle_{\Delta \omega, s}}$

Eqs. (10) and (11) use time and space averaged total energies of the subsystems divided by the mean power input:

$\eta_{i j}=\frac{1}{\omega} \frac{\left\langle E_{j i}{ }^{n}\right\rangle_{\Delta \omega, s}}{\left\langle E_{i i}{ }^{n}\right\rangle_{\Delta \omega, s} \cdot\left\langle E_{j j}{ }^{n}\right\rangle_{\Delta \omega, s}}$

The vibrational velocities $u k$ at any node $k$ of the subsystem can be computed with the FEM method. Total averaged energy $\langle E\rangle_{s}$ of subsystems $s$ in a frequency band $\Delta \omega$ is then obtained as follows:

$\langle E\rangle_{s}=\omega^{2} \sum_{k=1}^{N} m_{k}\left|u_{k}\right|^{2}$

Eq. (13) is accurate in the high frequency domain. This equation is not acceptable in the mid frequency domain, but has been used as a first step to estimate the total energies, though the RADIOSS software can provide the kinetic and potential energies, and consequently, an accurate estimate of the total energies.

\section{THE SEA-LIKE METHOD}

In the mid frequency domain, the conditions to get a proper SEA matrix are not satisfied for the following 
reasons: indirect coupling between non connected structures can occur, and the subsystems damping have a strong influence on the CLF, according to the SEA assumptions. At high frequencies, the intrinsic structural damping has no significant influence on power flow between subsystems. In the high frequency domain, the intrinsic damping becomes low. On the contrary, in the mid frequency domain, the damping has a great role on energetic transfer between subsystems. Consequently, in the mid frequency range, the damping cannot be separated from the coupling factors, as it is in SEA equations. In references [12] and [13], the Energetic Influence Coefficient EIC method is presented.

The approach consists, as in SEA, in considering $\mathrm{N}$ isolated structures (subsystems) and to assemble them, in order to constitute the complete structure. The eigenfrequencies and modes shapes of the total system are calculated. Under some assumptions [12]:

- The loads applied to subsystems $j$ and $l$ are uncorellated, if $j$ and $l$ are different,

- The loads applied to each subsystem are deltacorrelated,

- The space and time variables can be separated,

it is shown that the column matrix of subsystems kinetic energies is equal to the product of two matrices: a column matrix, the terms of which are the EIC, and a matrix of power spectral densities of forces applied to subsystems.

The SEA-Like method is very close to the EIC method, but the kinetic energy is replaced by the total energy.

The SEA-like approach can be combined with FEM in the same way as presented before. Direct FEM computations in the time or frequency domain can provide inputs to SEALike approach. In this case, all the subsystems are part of a Finite Element Model and the forced response of the whole system is computed with the FEM.

In the SEA-Like method, the subsystem total energies and input powers are related by:

$[E]=[A][P]$

where the A matrix is composed of Energy Influence Coefficients (EIC), $A_{i j}$.

$\left\{\begin{array}{c}E_{1} \\ \ldots \\ E_{n}\end{array}\right\}=\left[\begin{array}{ccc}A_{11} & \ldots & A_{1 n} \\ \ldots & \ldots & \ldots \\ A_{n 1} & \ldots & A_{n n}\end{array}\right]\left\{\begin{array}{c}P_{1} \\ \ldots \\ P_{n}\end{array}\right\}$

By exciting numerically the subsystems one by one, the following matrix system is obtained:

$\left[\begin{array}{cccc}\left\langle E_{11}\right\rangle & \left\langle E_{12}\right\rangle & \ldots & \left\langle E_{11}\right\rangle \\ \left\langle E_{21}\right\rangle & \left\langle E_{22}\right\rangle & \ldots & \ldots \\ \ldots & \ldots & \left\langle E_{k k}\right\rangle & \ldots \\ \left\langle E_{n 1}\right\rangle & \ldots & \ldots & \left\langle E_{n n}\right\rangle\end{array}\right]=\left[\begin{array}{cccc}A_{11} & A_{12} & \ldots & A_{1 n} \\ A_{21} & A_{22} & \ldots & \ldots \\ \ldots \ldots & \ldots & A_{k o t} & \ldots \\ A_{n 1} & \ldots & \ldots & A_{n n}\end{array}\right] *\left[\begin{array}{cccc}1 & 0 & \ldots & 0 \\ 0 & 1 & \ldots & \ldots \\ \ldots & \ldots & \ldots & \ldots \\ 0 & \ldots & \ldots & 1\end{array}\right]$

Consequently, the EIC $A_{i j}$ can be computed:

$$
\left[\begin{array}{cccc}
A_{11} & A_{12} & \ldots & A_{1 n} \\
A_{21} & A_{22} & \ldots & \ldots \\
\ldots & \ldots & A_{k t o t} & \ldots \\
A_{n 1} & \ldots & \ldots & A_{n n}
\end{array}\right]=\left[\begin{array}{cccc}
\left\langle E_{11}\right\rangle & \left\langle E_{12}\right\rangle & \ldots & \left\langle E_{1 n}\right\rangle \\
\left\langle E_{21}\right\rangle & \left\langle E_{22}\right\rangle & \ldots & \ldots \\
\ldots & \ldots & \left\langle E_{k k}\right\rangle & \ldots \\
\left\langle E_{n 1}\right\rangle & \ldots & \ldots & \left\langle E_{n n}\right\rangle
\end{array}\right]
$$

At high frequencies, the following matrix relation has to be asymptotically satisfied:

$\left[\begin{array}{cccc}A_{11} & A_{12} & \ldots & A_{1 n} \\ A_{21} & A_{22} & \ldots & A_{2 n} \\ \ldots & \ldots & A_{j j} & \ldots \\ A_{n 1} & \ldots & \ldots & A_{n n}\end{array}\right]=\frac{1}{\omega}\left[\begin{array}{cccc}\sum \eta_{1 i} & -\eta_{21} & \ldots & -\eta_{n 1} \\ -\eta_{12} & \sum \eta_{2 i} & \ldots & \ldots \\ \ldots & \ldots & \sum \eta_{k i} & \ldots \\ -\eta_{1 n} & \ldots & \ldots & \sum \eta_{n i}\end{array}\right]^{-1}$

This equation is written:

$[A]=\frac{1}{\omega} *[\eta]^{-1}$

This approach has been performed using the classical modal analysis by C. R. Fredö [10], and B. Mace [5]. B. Mace has shown the influence of the modal overlap on the coupling terms and has pointed out the cases in which the indirect CLF need to be taken into account. It is important to note that, in the methodology developed in this paper, the SEA-Like (mid frequency predictions) subsystems and the SEA (high frequency prediction) subsystems are the same.

\section{LOADING OF THE STRUCTURAL FINITE ELEMENT MODEL}

The main difficulty of the methodology lies in the loading of the structure. The question is to know how to excite the structural Finite Element Model to obtain accurate CLF and EIC for energetic methods of concern, such as SEA and SEA-Like respectively. It has been chosen to apply to the Finite Element Model 'rain on the roof' excitations, to estimate unknown quantities. The 'rain on the roof' loading consists in impulse forces randomly distributed both in time and space over the surfaces of the subsystems defined as follows in the time domain:

$F=\sum_{i=-\infty}^{\infty} f_{i}\left(t-t_{i}\right) \delta\left(x-x_{i}\right)$

$f_{i}\left(t-t_{i}\right)$ gives the time dependency of the force during the impact with $t_{i}$ the peak time of the $i$-th impact, and $x_{i}$ the location of the $i$-th impact. In the frequency domain, the 'rain on the roof' excitation is represented by a white noise, with random relative phases, applied to nodes of the mesh. This loading is compatible with SEA deep assumptions: the forces applied to subsystems have to be steady, random and of constant spectral densities. Of course, 'rain on the roof' excitation is very far away from real excitations encountered during launch vehicles flights. The problem of taking into account real excitations, such as aero-acoustic excitations is under investigations $[14,15]$.

This paper is here focused on the accurate application of 'rain on the roof' excitation for needs of the energetic 
methods of interest, SEA and SEA-Like, in the case of flexural loading. The number of 'rain of the roof' loads to be applied to the subsystems, and the distribution of the 'rain of the roof' loads on a subsystem in order to excite all modes of the subsystems are the key problems to solve. The number of 'rain on the roof' forces required to excite all modes of the structures in the frequency band of concern, which is the main point of discussion, is not discussed a lot in the open literature. D. A. Bies [7], has written that a number of three loads per subsystem is sufficient to excite the modes. A more objective criterion, "Influence Circle" criterion [4], is proposed here for defining the number of excitations points.

\section{A. "Influence Circle" Method}

The key point is to define a loading, which excites all flexural modes. As mentioned before, the loads are punctual, and spatially and temporally uncorrelated. The main question is the number of point loads to be applied to the structure to excite all flexural modes. The idea is to estimate the area of the structural zone influence by a point load. Let us consider a circular plate subject to a perpendicular point force. The solution of the flexural motion equation of this plate subject to a point force is a Bessel function, which has a pseudoperiod [16]. Let us define the spatial influence zone of this point load as the value of this pseudo period. The radius $\rho_{\text {inf }}$ of the circle of influence, which depends on the frequency, is defined as follows [16]:

$$
\rho_{\mathrm{inf}}=\frac{\pi}{\sqrt{\omega \sqrt{\frac{\rho_{s}}{D}}}}
$$

This formulation is valid at high frequencies where the surface of the plate are much higher the surface of the influence zone. In the case of circular plate of radius a, it leads: $\frac{\pi}{\omega \sqrt{\frac{\rho_{s}}{D}}} \leq a^{2}$. It follows: $\omega \geq \frac{\pi}{a^{2} \sqrt{\frac{\rho_{s}}{D}}}$

The idea is to determine the optimal number of loads nbloads $_{\text {optim }}$ by dividing the subsystem total surface $S_{\text {plate }}$ by the spatial influence zone, called "influence circle" surface:

nbloads $_{\text {optim }} \times 2 \pi \rho_{\text {inf }}=S_{\text {plate }}$

It is not sufficient to define the optimal number of point loads to excite all structural flexural modes. The point loads have to be uniformly distributed all over the surface of the subsystem to be excited. The distribution of the point loads over the surface is defined using the OLH method.

\section{B. OLH Loads Distribution}

In order to achieve a set of of 'rain on the roof' excitations to the FEM model which excites all modes of the structure, we use an OLH (Optimal Latin Hypercube) scheme (Hyperstudy/Dss sampling module [17]). Classically, a "Monte Carlo" simulation is used to obtain a random distribution ( $\mathrm{N}$ stochastic variables, ie coordinates) of the samples which are chosen from their own distribution independently from each other. This method is not fully satisfactory and does not provide a coverage of all excitation modes. The main drawback [18], is that samples may be unevenly distributed, very close to each other in one region and not enough density elsewhere.

The purpose of $\mathrm{LH}$ is to divide space into regions of equal probability (squares if the number of variable is 2 , cubes if 3 ) depending on the desired number of samples. The LH algorithm makes sure that all regions have at least one sample. The OLH method adds an algorithm that allows, by measuring distances, an optimal repartition among a number of LH distributions, thus ensuring that not only the coverage is evenly distributed, but that it is also optimal in the "geographic" sample positions selected.

The definition of an OLH distribution is a two steps process. The first one consists in defining $\mathrm{p}$ stochastic variables (with given distributions) as inputs to characterize the loaded subsystem surface geometry (lengths, or radius for instance). Once the OLH computation is done, the output leads to $\mathrm{N}$ excitations locations, where $\mathrm{N}$ is the number of samples. Clustered samples situation is avoided using descriptive sampling, which consists of setting the grid. Each square (if $p=2$ ) has equal probability. Final point coordinates are the centers of these areas. Therefore the problem becomes discrete and will be solved using optimized OLH. Calculation of OLH is only dependant on the number of samples $(\mathrm{N})$ and the number of variables $(\mathrm{p})$ : Considering points $\left\{X_{i}\right\}_{i=1}^{N}$ with integer coordinates in the interval $[1, \ldots, N]$ in $\mathrm{p}$ space dimensions. The coordinates of the points can be arranged in the following L-matrix:

$L=\left(\begin{array}{c}X_{1} \\ \vdots \\ X_{N}\end{array}\right)=\left(\begin{array}{ccc}x_{11} & \cdots & x_{1 p} \\ \vdots & & \vdots \\ x_{N 1} & \cdots & x_{N P}\end{array}\right)$

The points constitute a $\mathrm{LH}$ if each column in $\mathrm{L}$ is a permutation of $\{1, \ldots, N\}$. For OLH, two criteria can be used to describe the optimal repartition:

The first is the minimum distance between points:

$d(L)=\min _{1 \leq i, j \leq N, i \neq j}\left\|X_{i}-X_{j}\right\|$

where $\|$.$\| represents the Euclidean norm of a vector.$ Between two samples L1 and L2, the highest minimum distance is kept (if $d(L 1)>d(L 2)$ then $\mathrm{L} 1$ is better than L2).

The second is the total number of times a minimum distance occurs is taken into account, denoted $\mathrm{n}(\mathrm{L})$, Between two samples L1 and L2 which have the same minimum distance, $\mathrm{L} 1$ is considered better if $n(L 1)<n(L 2)$.

The process to generate a random $\mathrm{LH}$ is as following. The first column of the L-matrix is chosen to $\{1, \ldots, N\}$. Then, to generate each of the remaining columns, the process is as follows: the new element is chosen randomly among $\{1, \ldots, N\}$, same for next element, but this one is checked to be not the same as the previous elements in the same 
column. If this one is different, it is used; otherwise a new number is taken among $\{1, \ldots, N\}$ and again compared with previous ones. To illustrate on a $\mathrm{p}=2$ ( 2 dimensions) example with $\mathrm{N}=4$ samples. The grid is set by dividing both axis in four equal probability zones. A sixteen cells grid is obtained. The L-matrix first column is completed as following:

$L=\left(\begin{array}{l}1 \ldots \\ 2 \ldots \\ 3 \ldots \\ 4 \ldots\end{array}\right)$

On the second column, the element to be randomly chosen can be $1,2,3$ or 4 , the next one is also randomly picked among 1, 2, 3 and 4, but will be kept only if different from the previous elements of the same column. In the end, the L matrix is completed, for example as below:

$L=\left(\begin{array}{ll}1 & 3 \\ 2 & 2 \\ 3 & 4 \\ 4 & 1\end{array}\right)$

Here is a random LH sampling. These coordinates are mapped using the real variables dimensions and the type of random distribution to obtain the real coordinates. For OLH, a step for distance comparisons is added before the mapping step. Figs. (1-3) compare the three distributions with hundred samples each. The first chart shows a Monte Carlo distribution, the middle one stands for a $\mathrm{LH}$ and the last for OLH. This last one is the best solution to obtain an optimal stochastic repartition for "rain on the roof" excitation modeling.

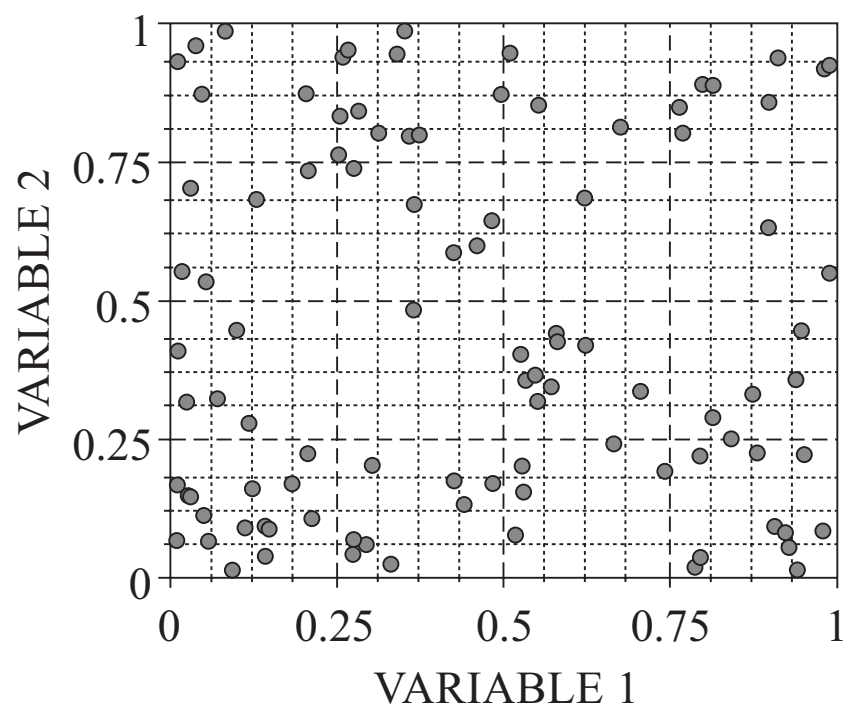

Fig. (1). Monte Carlo stochastic distributions of a 100 points sample for 2 variables.

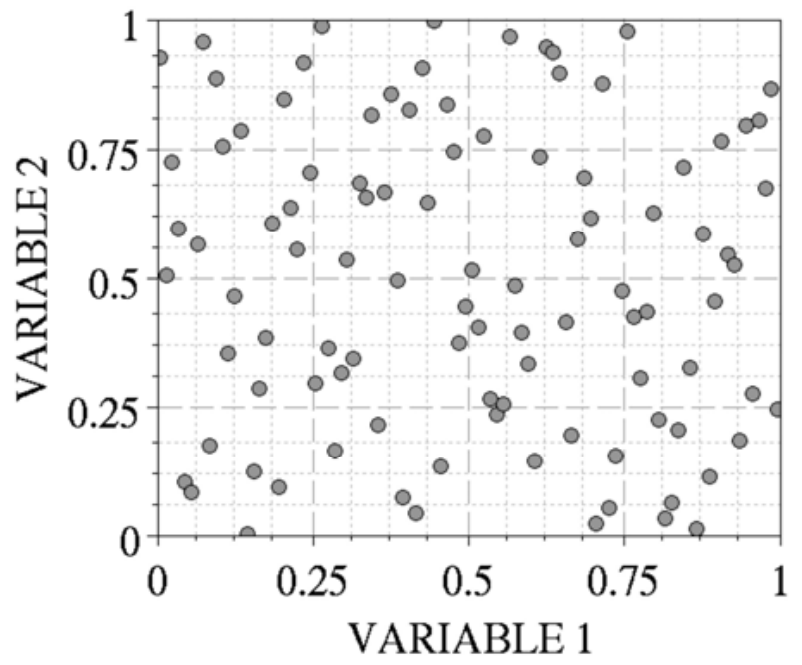

Fig. (2). LH stochastic distributions of a 100 points sample for 2 variables.

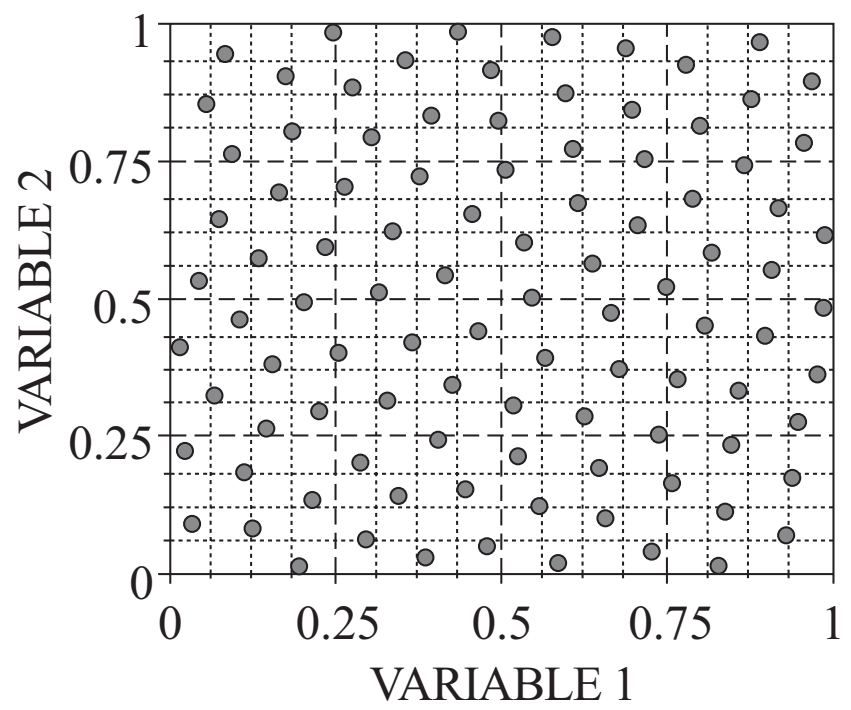

Fig. (3). OLH stochastic distributions of a 100 points sample for 2 variables.

\section{APPLICATION TO ACADEMIC CASES}

Two types of academic cases have been considered in this paper: a two plate case and a three plates case.

\subsection{Two Plates Case}

The first step of the feasibility study of purely numerical PIM approach is performed on a simple case, for which the coupling loss factors CLF can be estimated by closed formulations. The example consists of two aluminum plates with the same thickness and coupled by a line junction, as shown in Fig. (4).

The total energy of a subsystem is assumed to be equal to two times the kinetic energy. This hypothesis is reasonable at high frequencies, frequency range where the PIM method works. 
The coupling between subsystems flexural modes of these two coupled plates is here investigated. The validity of the purely numerical PIM is investigated by comparing the results provided by the numerical PIM to closed formulation established by R. H. Lyon and E. Eichler [6], for the flexural coupling loss factor between plates perpendicularly connected. In [6], the Coupling Loss Factor is obtained using a waves approach. It is obtained analytically by considering the subsystems as semi-infinite. The vibrational field is assumed to be diffuse in the subsystems, and it is represented by incoherent plane waves. The Coupling Loss Factor is then obtained by estimating the transmission coefficient between subsystems, which is averaged over all angles of incident plane wave $[6,7]$. In the case of two coupled plates, the analytical CLF is expressed as follows:

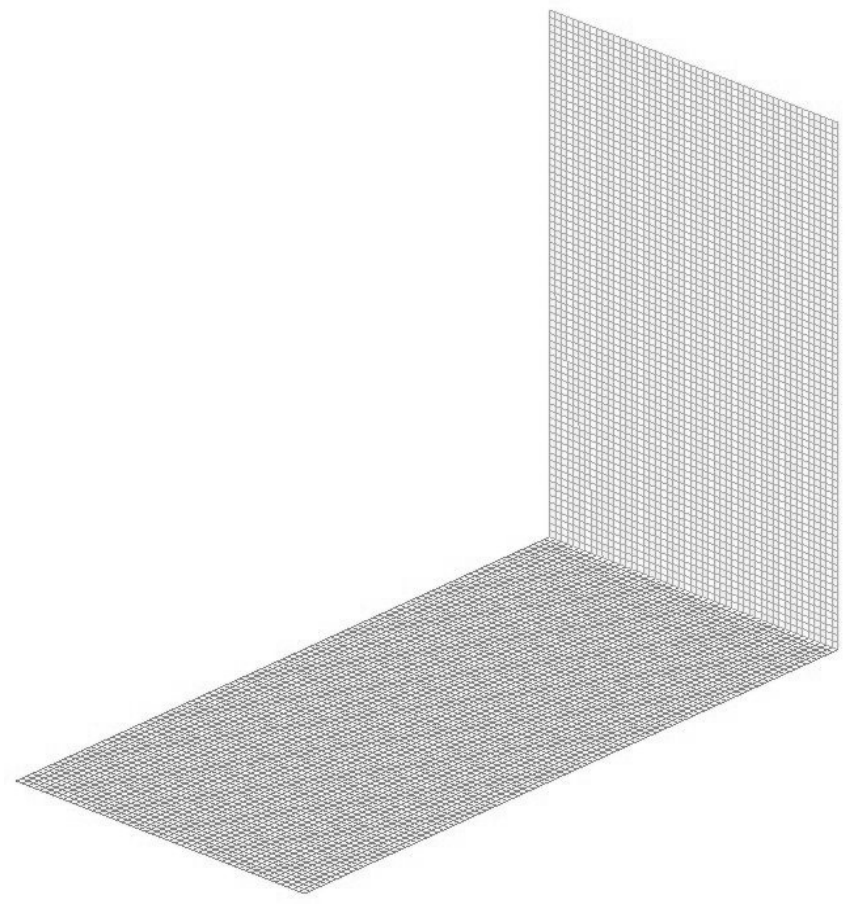

Fig. (4). Academic case: « Two Plates » Finite Element Model.

$\eta_{i j}=\frac{k_{b} L_{i j} \tau_{i j}}{\pi A_{i}}$

For two coupled plates manufactured in an identical material and having the same thickness, an analytical approach can provide the mean transmission coefficient [6]

$\tau_{i j}=\frac{1}{3}$.

The hypothesis of diffuse field implies that the subsystems have a sufficient number of resonant incoherent modes in the frequency band of interest. The dimensions of the plates are chosen in order to have a relatively large modal density over all third octave frequency bands of interest. The junction is $0.5 \mathrm{~m}$ long and the lengths of the plates are respectively $0.75 \mathrm{~m}$ and $1 \mathrm{~m}$ for the two subsystems. This Finite Element Model has more than 5 modes per frequency band for the two subsystems over the whole frequency range of interest: from $200 \mathrm{~Hz}$ to $2500 \mathrm{~Hz}$, which allows the use of energetic methods. L. D. Pope et al. has written that 7 modes per one third octave bands is required to ensure a sufficient modal overlap $[19,20] .5$ modes is consequently the lower limit.

The mesh of the two plates has been defined using a classical wave length criterion. The Finite Element Model is realized in order to estimate the forced response of the structure for a frequency range from $100 \mathrm{~Hz}$ to $3500 \mathrm{~Hz}$. The wave number criterion used here takes the form of $l_{\text {mesh }} \leq \lambda / 5$, which is a reasonable criterion in the case of steady state excitations. This criterion leads, for our case of interest, to a number of 8575 quadrilateral shell elements, (Fig. 4). Because of the difficulty to realize simply supported boundary conditions in an experimental study, free-free boundary conditions have been chosen in order to allow comparison with experimental data.

The forced response of the complete structure can be estimated by different approaches, the classical modal approach, widely used in the low frequency domain for industrial problems, the direct frequency domain resolution, which uses a direct inversion of the dynamic stiffness matrix in the frequency domain, the time domain resolution, using explicit analysis. The modal approach is faster, but leads to large errors on the structural natural frequencies in the mid and high frequency domains.

Explicit codes allow computations at higher frequencies than implicit codes with a reasonable computation time, we have chosen to perform calculations in the time domain using the RADIOSS software of Altair Development France. Calculations in the time domain using the RADIOSS software have already been carried out [14]. We have chosen here to use the numerical PIM validation by performing computations in the time domain, using an explicit solver (RADIOSS software). FEM computations provide, among all possible results, velocities at nodes. According to [15], the normalized total energies of subsystems are computed, and the Lalor formula described in Eq. (11) is used to obtain CLF values to be used in SEA analysis.

In the hybrid FEM/SEA approach, the usual high frequency SEA decomposition into subsystems is kept. The CLF are quantified, to be used in the SEA analysis. The expression of the power exchanged between subsystems is obtained in classical SEA under several hypotheses regarding the nature of the excitation, which have to be random and broadband.

\subsubsection{Influence of the Number of Loads and their Distribution}

In this feasibility study, the excitations are "rain on the roof' excitations, defined using the "Influence Circle" method and the OLH distribution. To illustrate the results improvements using these methods, two analyses have been made, the analysis of the influence of the increasing number of the excitation loads up to the optimal number provided by the "Influence Circle" method, and the influence of the loads random distribution, by comparing results provided by OLH and by Monte-Carlo methods. For the "two plates" model, the optimal number of loads per plate has been calculated 
and is 19 on the first plate and 25 on the second plate for the computation of the highest frequency.

The computation cases will be noted as follows: Model \# a $(\mathrm{x}, \mathrm{y})$, where $\mathrm{a}$ is an indice for the computation case, $\mathrm{x}$ denotes the number of point loads applied to plate 1 , and $y$, the number of point loads applied to plate 2 . Consequently, this computation case is denoted Model \# 4 (19; 25). Five other couples of number of loads have been investigated, from $(5 ; 7)$ loads up to $(38 ; 50)$. For each of them, a distribution of random loads was obtained with the OLH algorithm. Fig. (5) displays the six Finite Element Models with the loads repartitions (arrows). Fig. (6) displays CLF values obtained by numerical PIM computation between two plates against third octave frequency bands; from $100 \mathrm{~Hz}$ to $3200 \mathrm{~Hz}$. Closed formulation values of CLF, obtained using Eq. (19), are plotted in bold red. Focusing on mid-high frequency results (Fig. 6), from third octave band of central frequency $250 \mathrm{~Hz}$, it becomes that, when models are sufficiently excited, i.e. from $(19 ; 25)$ loads, a significant improvement of correlation occurs between numerical and closed formulation of CLF values. Fig. (7) shows the loads distribution over "Two Plates" model resulting from MonteCarlo and OLH computations (number of loads are the same for each model). Fig. (8) displays numerical PIM results of CLF values between two plates, depending on loads distribution over the surface of the plates, compared to CLF closed formulation values. Green curve is for CLF obtained with loads distribution using a Monte-Carlo scheme, brown curve obtained with OLH scheme, bold red line is for closed formulation values. These results show a better agreement with analytical values, when OLH scheme is used to define the point loads distribution.

\subsubsection{General Interpretations}

Fig. (9) shows a satisfactory agreement between the numerical PIM and closed formulation in the high frequency domain ("model\#4" is the computation case using optimal number and distribution of loads provided by the "Influence Circle" method and the OLH scheme). The error is less than 4 dB from $200 \mathrm{~Hz}$ up to $3500 \mathrm{~Hz}$. Below the third octave band of central frequency $250 \mathrm{~Hz}$, significant discrepancies between the computed values and the analytical results are observed. In those frequency bands, each plate has a low number of modes and the modal overlap becomes too low to have a proper SEA model. Furthermore, the hypothesis of diffuse field for the application of CLF analytical formulation described at Eq. (27) is not valid. The comparison between the two approaches can not, consequently, lead to conclusions on the validity of the hybrid FEM/SEA approach. In the mid-frequency range (100 Hz to $200 \mathrm{~Hz}$ ), PIM method, which is based on SEA, is not available, but SEA-Like approach provides accurate results.
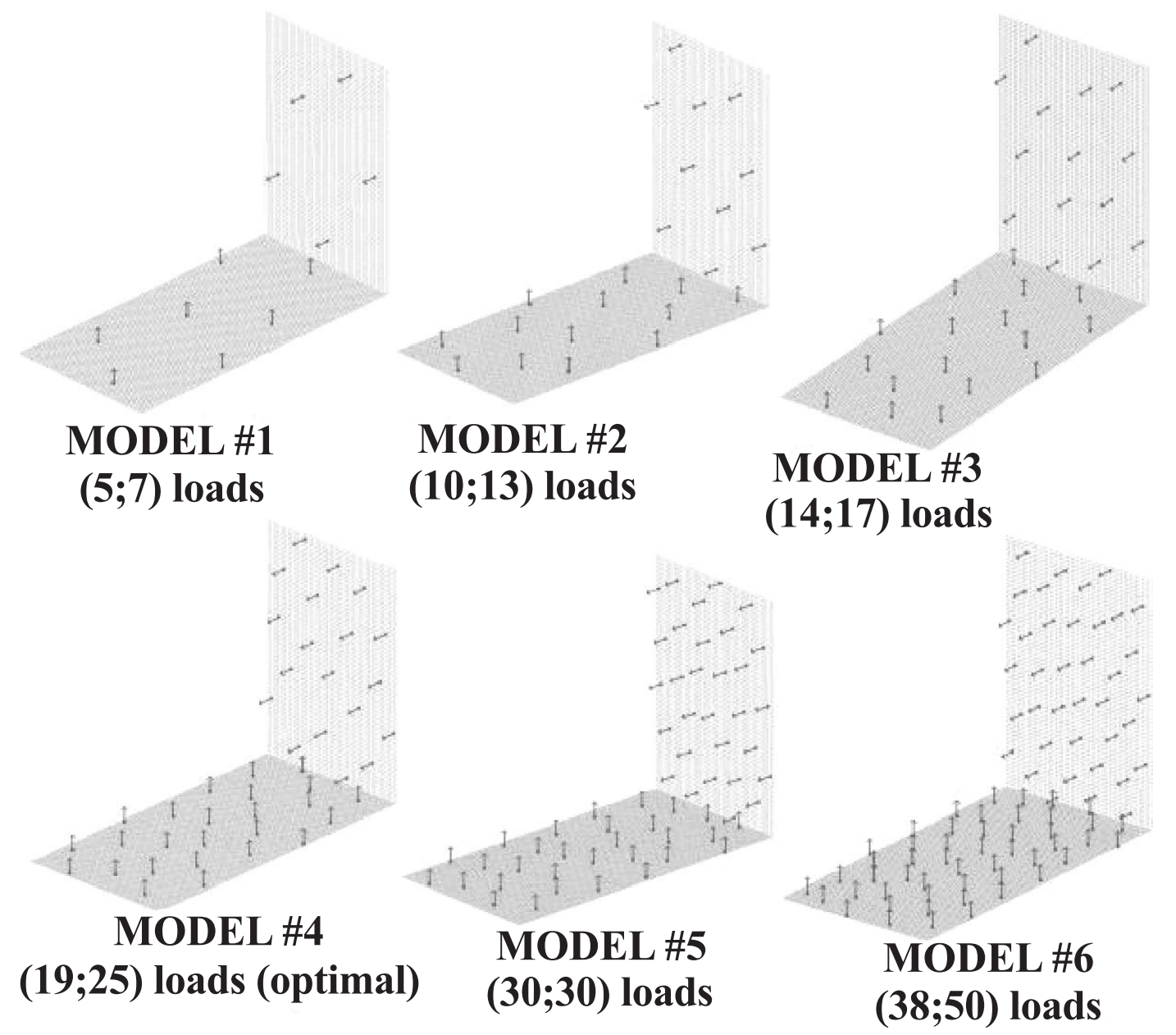

Fig. (5). Plates academic case. Computation cases with different point loads numbers. 


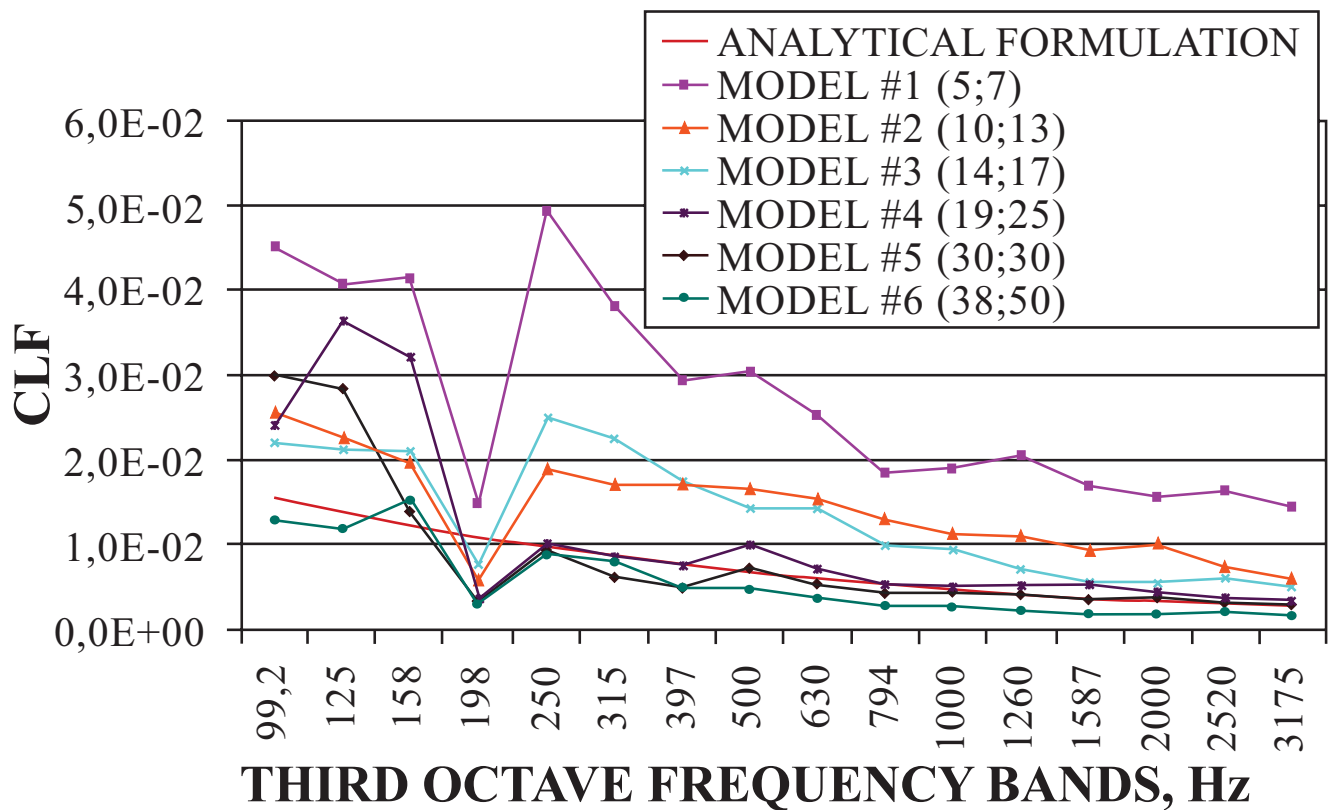

Fig. (6). Plates academic case. Computed CLF values versus the point loads numbers.

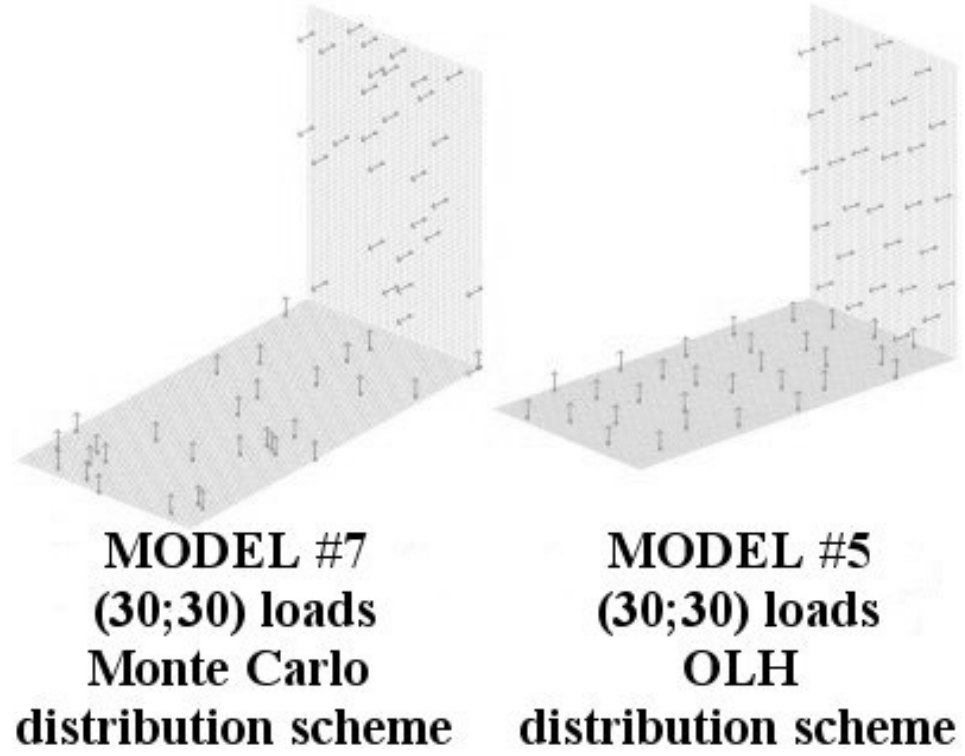

Fig. (7). Two plates academic case. Distribution of 30 point loads per subsystem using Monte Carlo and OLH distribution schemes.

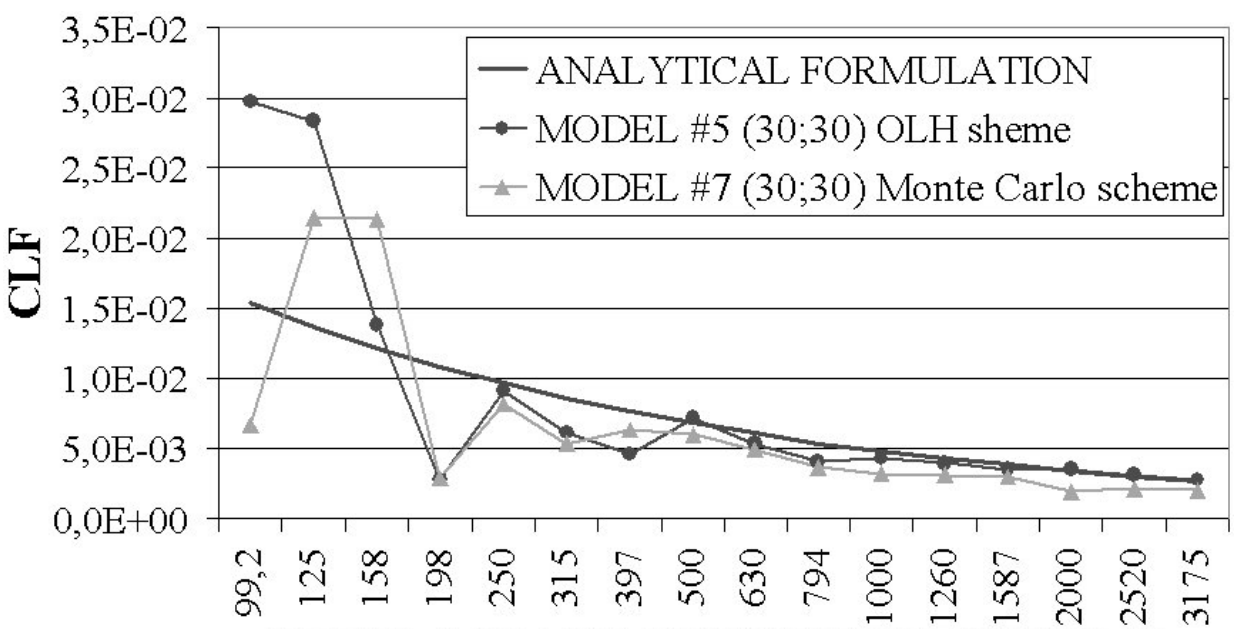

THIRD OCTAVE FREQUENCY BANDS, $\mathrm{Hz}$

Fig. (8). Two plates academic case. CLF values depending on point loads distribution. 


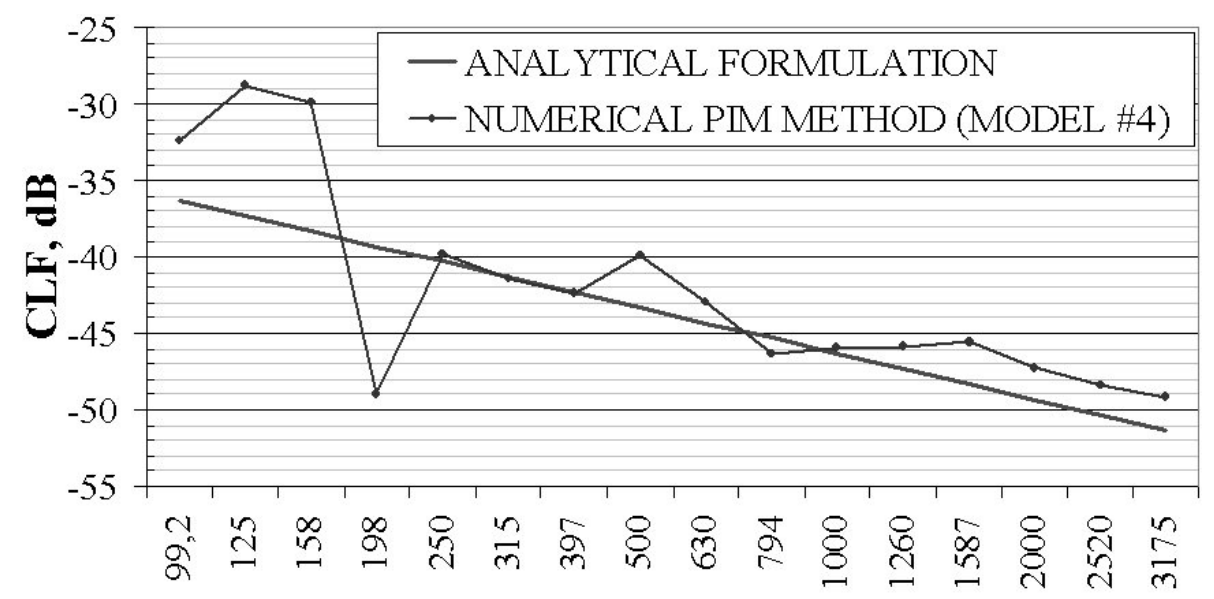

THIRD OCTAVE FREQUENCY BANDS, Hz

Fig. (9). Two plates academic case. CLF values comparison between analytical formulation and numerical PIM results.

\subsection{Three Plates Case}

A three plates configuration has been investigated as a further step toward industrial cases. The reason why the three plates case is investigated is that this case allows the analysis of the indirect coupling.

This model (Fig. 10) is identical to the two plates model, but another $(0.5 \mathrm{~m} \times 1 \mathrm{~m})$ plate has been added, symmetrically, to the $0.5 \mathrm{~m} \times 0.75 \mathrm{~m}$ ) plate. Excitations are applied to the plates, as explained before, by using the Influence Circle criterion and OLH process.

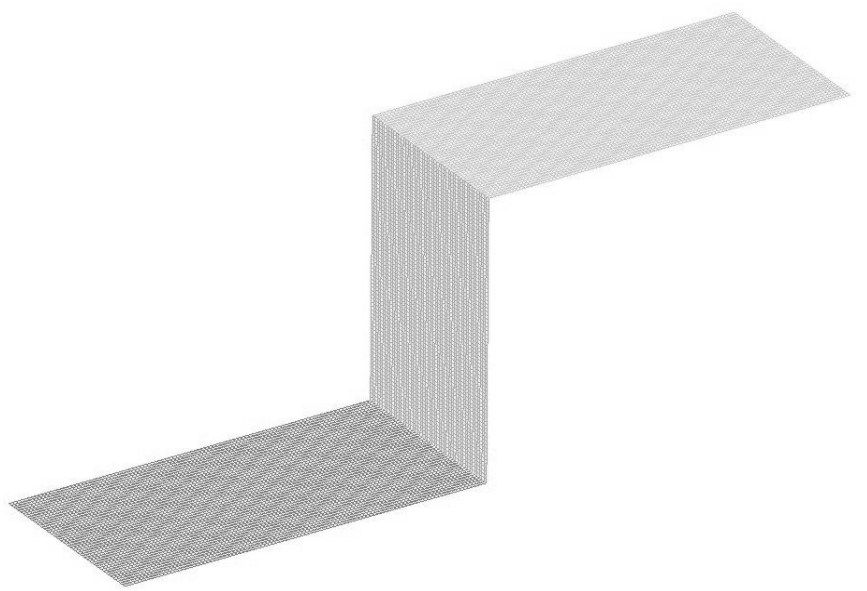

Fig. (10). View of the « Three Plates » Finite Element Model.

The Finite Element Model computations provide, among all results, the velocities of each node of the model. Energy and input power are computed to raise $\left[\left\langle E_{i j}\right\rangle\right]$ matrix, which is inversed to obtain CLF matrix as in Eq. (8). Fig. (11) shows CLF values (between subsystems "1" and "2") obtained in the case of "Two Plates" and "Three Plates" models versus analytical plates CLF formulation. The results are quite different between CLF values computed for the cases of "Two Plates" and "Three Plates" models, whereas analytical formulation, for same input data (two plates are coupled), provides, of course, the same results.
At high frequencies, these discrepancies are small and acceptable, but are more important in the mid-frequency range. Of course, due to the third plate presence, global modes indirect coupling effects occur.

\subsection{Validation of SEA-Like Method}

To validate SEA-Like results in the mid frequency range, we know from Eq. (18) that EIC matrix must asymptotically converge to the inverse CLF matrix divided by the radian frequency according to Eq. (19). As a validation criterion, to point out this convergence, the CLF matrix is obtained using PIM method and Lalor Eq. (10). Diagonal terms of CLF matrix are then classically found with the following relation:

$$
\sum \eta_{j i}=\eta_{j}+\sum_{i \neq j} \eta_{j i}
$$

and:

$\eta_{j}=\frac{1}{\omega} \frac{1}{\left\langle E_{j j}\right\rangle}$

Four representative curves are displayed on Figs. (12-15) comparing EIC and terms of right side of Eq. (19) obtained by the numerical PIM. The plotted curves have the same dependency with frequency, but do not tend to each other for high frequencies, remaining parallel. The computations have been performed not at frequencies high enough so that the convergence can be observed.

We can say however that the convergence criterion is satisfied. We may note as a result that indirect coupling is negligible in this model, therefore SEA-Like and numerical PIM provide very close results.

\section{APPLICATION TO AN INDUSTRIAL CASE}

\subsection{Vehicle Equipment Bay (VEB) Finite Element Model}

In order to perform a industrial feasibility study of SEALike method, the SEA-Like approach has been applied to the Vehicle Equipment Bay (VEB) of the ARIANE 5 Launch Vehicle. The ARIANE 5 VEB is divided into five subsystems, as shown in Fig. (16). Excitation forces have 


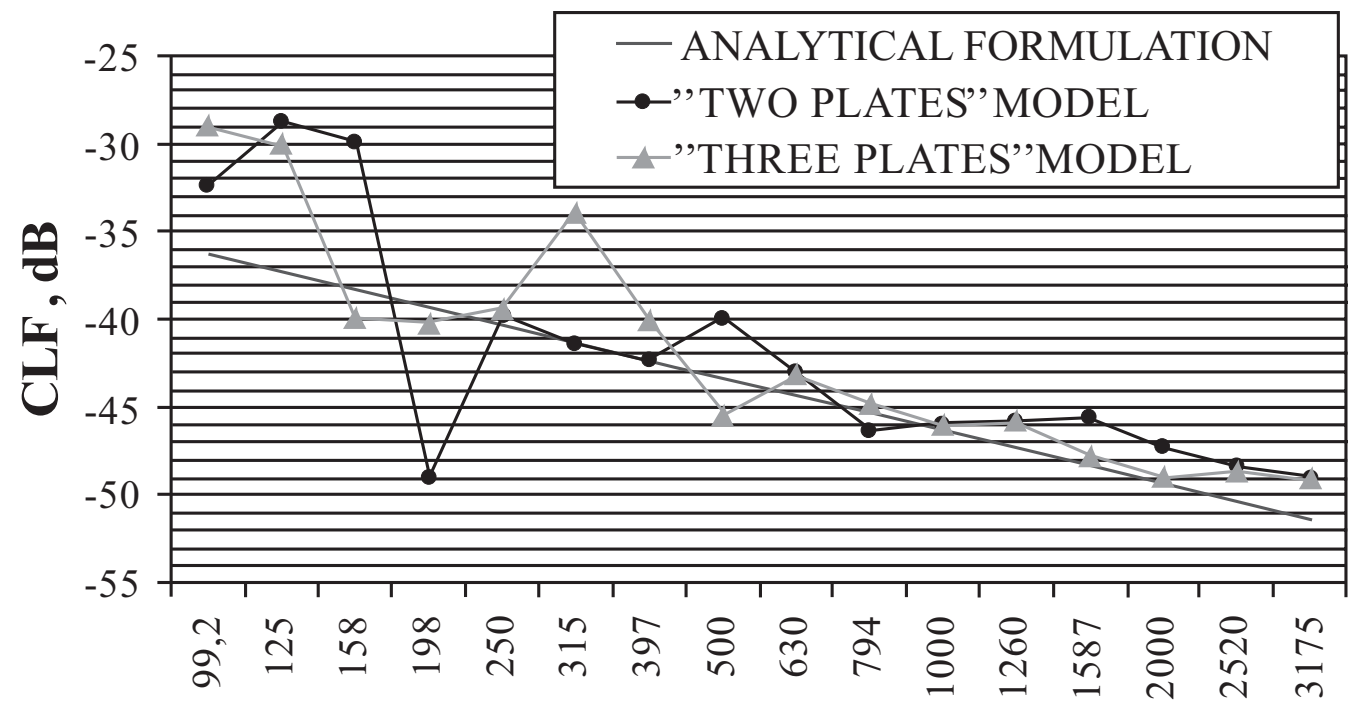

THIRD OCTAVE FREQUENCY BANDS, Hz

Fig. (11). Three Plates and Two Plates Academic Case. Comparison between CLF obtained from closed formulation and CLF using numerical PIM for « Two Plates » and « Three Plates » models.

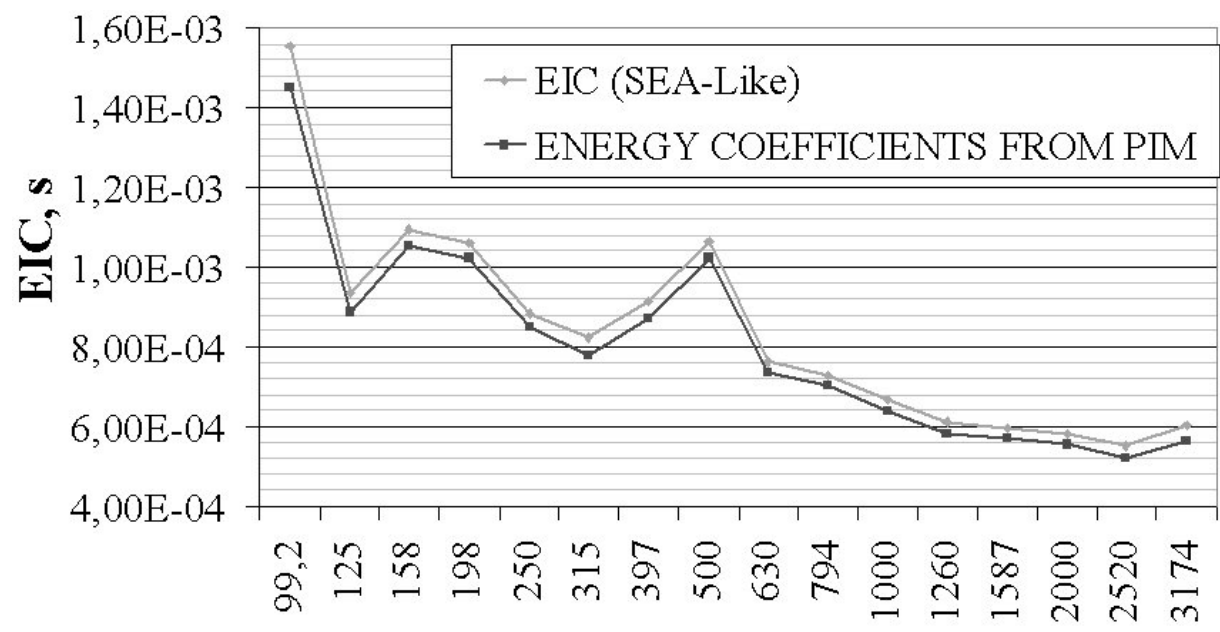

\section{FREQUENCY THIRD OCTAVE BANDS, Hz}

Fig. (12). Energy Influence Coefficients from SEA-Like versus Energy coefficients computed with numerical PIM according to the equation 19: results of plate 1 when plate 1 is loaded.

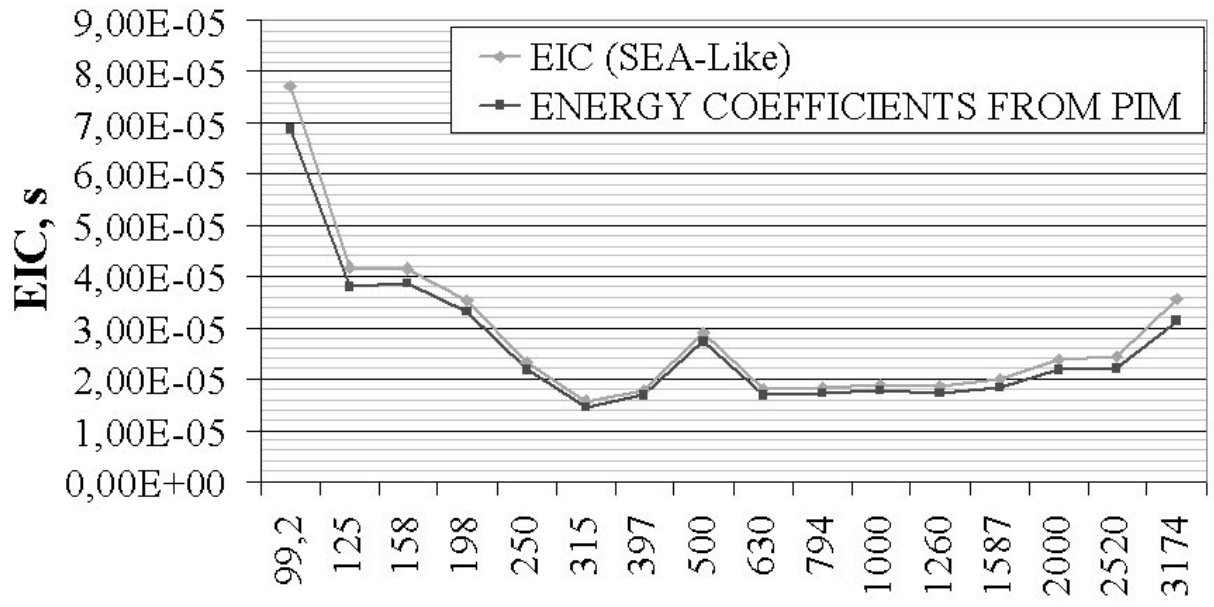

\section{FREQUENCY THIRD OCTAVE BANDS, Hz}

Fig. (13). Energy Influence Coefficients from SEA-Like versus Energy coefficients computed with numerical PIM according to the equation 19: results of plate 2 when plate 1 is loaded. 


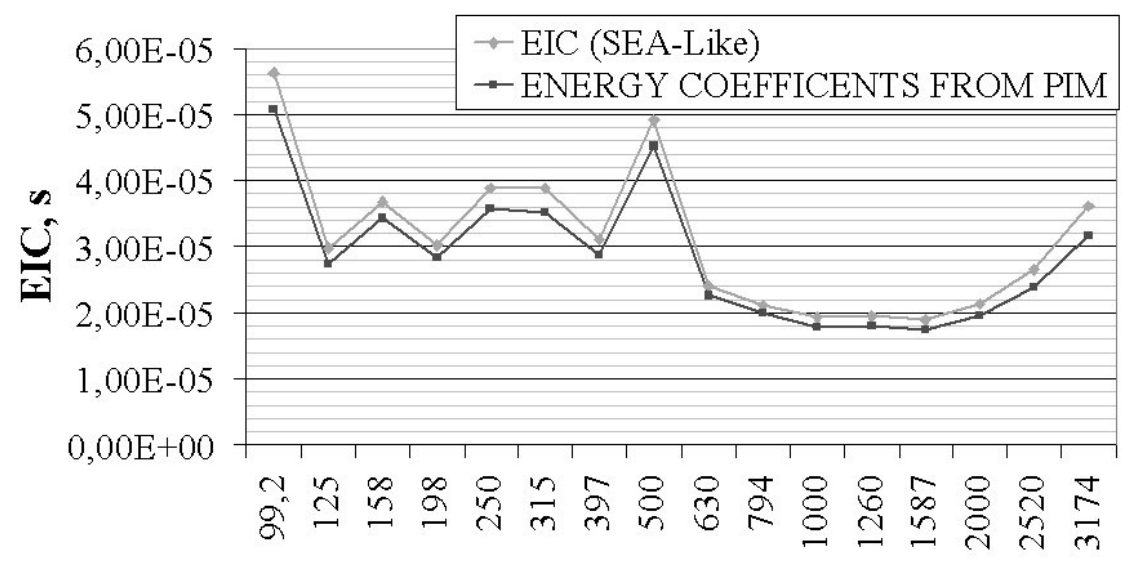

\section{FREQUENCY THIRD OCTAVE BANDS, Hz}

Fig. (14). Energy Influence Coefficients from SEA-Like versus Energy coefficients computed with numerical PIM according to the equation 19: results of plate 3 when plate 1 is loaded.

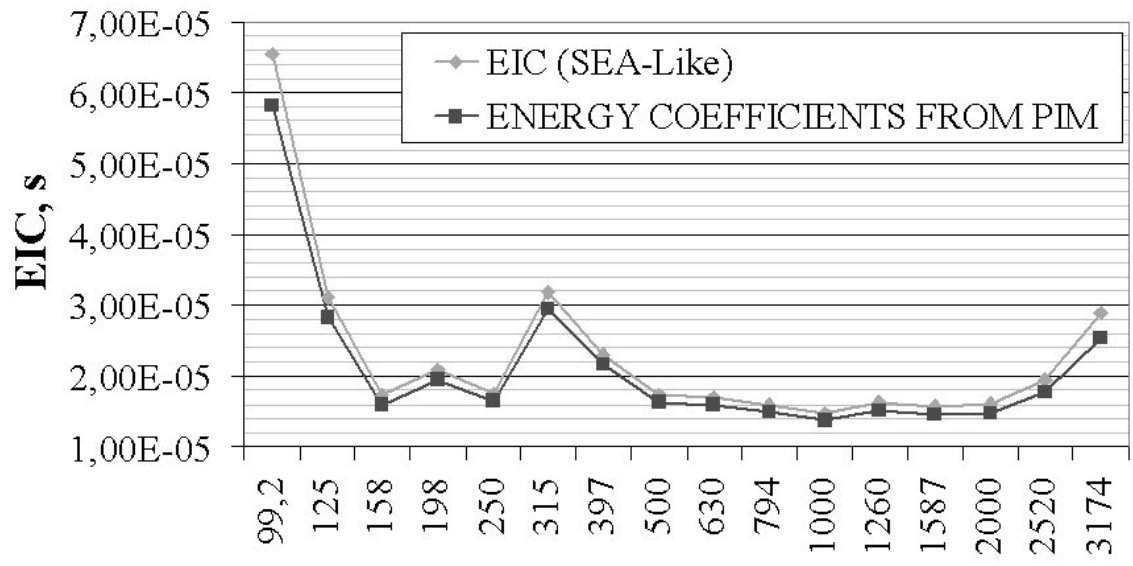

\section{FREQUENCY THIRD OCTAVE BANDS, Hz}

Fig. (15). Energy Influence Coefficients from SEA-Like versus Energy coefficients computed with numerical PIM according to the equation 19: results of plate 1 when plate 2 is loaded.

been applied to 30 nodes per subsystem. In this approach, structural subsystems are structural parts between physical boundary conditions. Making this, all structural modes are taken into account in the computations. Dividing a structural part between physical boundary conditions into a greater number of subsystems would lead to a truncation of the modal basis: the global modes would be lost.

The loads are "rain on the roof" loads, spatially and temporally non-correlated. In order to obtain the locations, where the "rain on the roof" loads have to be applied to each
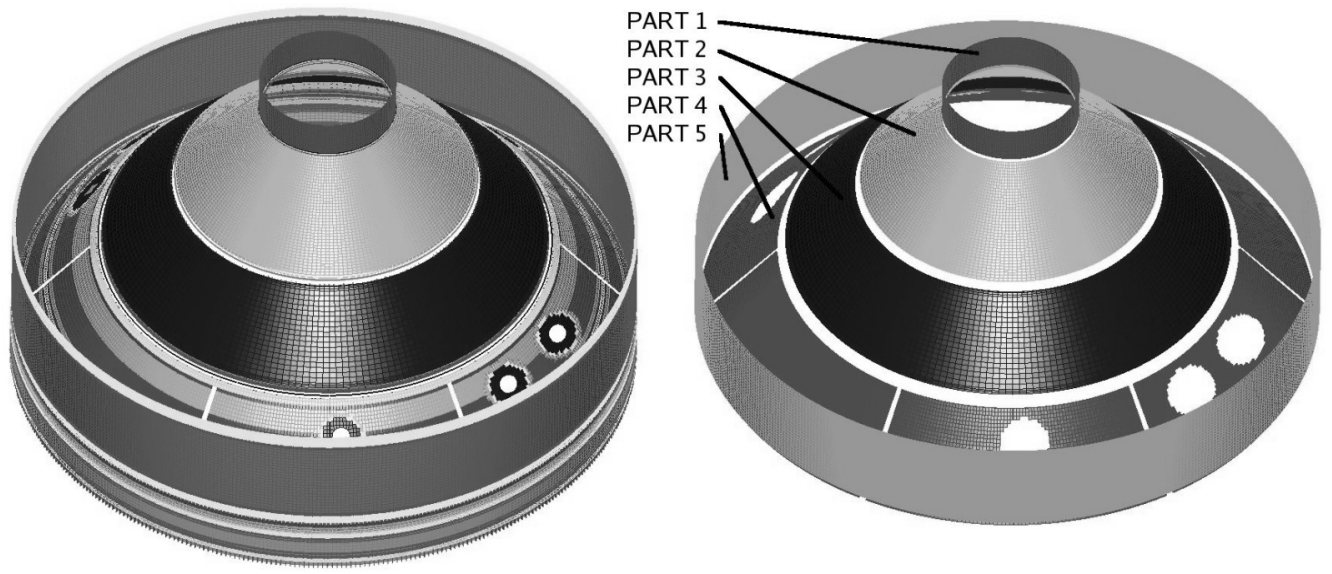

Fig. (16). On the left: ARIANE 5 VEB (industrial case) original Finite Element Model. On the right: ARIANE 5 VEB model decomposition into subsystems (PARTS) 


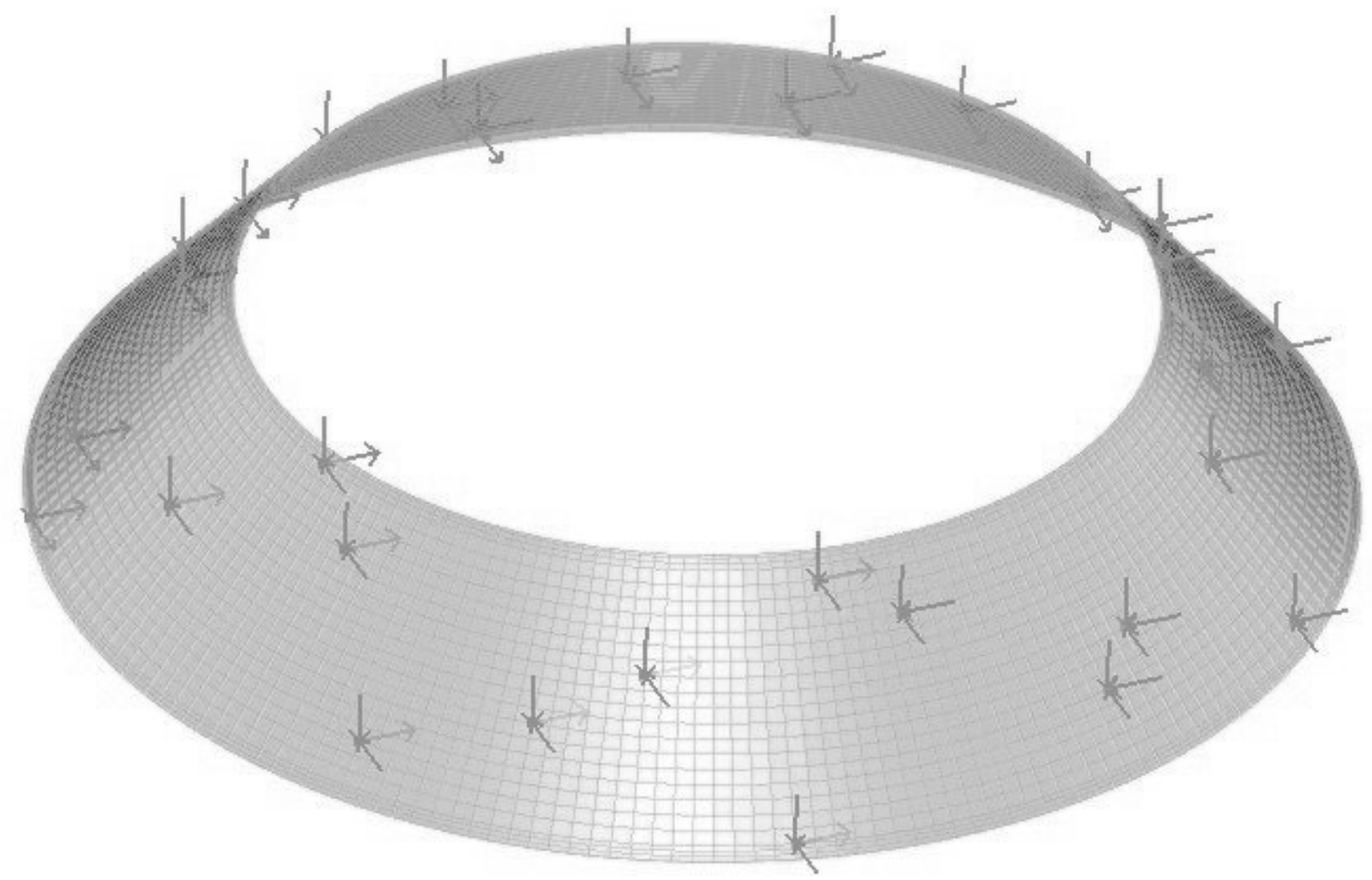

Fig. (17). Industrial Case. Point Loads Distribution on subsystem \#2 of the ARIANE 5 VEB Finite Element Model.

subsystem, as well as the computation duration, we have used an OLH distribution and applied the "Influence Circle" method, which ensure an optimized distribution of the excitation loads all over the subsystems surfaces, Fig. (17), and a sufficient number of point loads, respectively. Such load distribution is the key point to excite all flexural modes of the subsystems. The applied loads are Dirac excitations, as shown in Fig. (18). The computations are carried out in the time domain by using an explicit code. The duration of each computation is $51.2 \mathrm{~ms}$ and the output samples from the explicit code are stored every $0.1 \mathrm{~ms}$. This allows a correct coverage of the bandwidth of industrial interest, up to 5000 Hz. For each computation run, a different subsystem is loaded in order to compute EIC coefficients. For ARIANE 5 VEB, 5 computation runs have then been performed.

\subsection{CLF and EIC Extraction Process}

The computations are carried out according the following two steps: first a PIM estimate of the CLF between the structural subsystems is performed, and then a SEA-Like estimate of the EIC between the structural subsystems is conducted. After each computation run, a Fast Fourier Transform (FFT) of the velocities of all the nodes of each subsystem is carried out. The vibrational energies and injected powers of each subsystem are then calculated using respectively the following relation:

$E=M<v^{2}>$

where $\mathrm{M}$ is total mass of the subsystem and $\left\langle v^{2}\right\rangle$ is the mean of the squared nodal velocities. The injected power is computed from nodal velocities of nodes and from the injected force expressed in the frequency domain, according to the following equation:

$$
P_{i n j}=\operatorname{Re}(F \bar{V})
$$

For each run, once energies and injected powers are computed, they are spatially averaged over each part or subsystem of the structural model. They are then averaged per each one third octave band. After this, energies are normalized, dividing them by the injected power. Performing 5 computations run (five subsystems for the ARIANE 5 VEB case), a 5 by 5 matrix of total energies $\left[\left\langle E_{i j}\right\rangle\right]$ of Eq. (17) is consequently obtained. PIM analysis is used to extract the CLF using Eq. (8). In the SEA-Like analysis, the EIC matrix from Eq. (17) is directly obtained from normalized total energies matrix. To check the validity of this matrix, it is verified that the EIC converge to the energy coefficients from PIM, according Eq. (18) and Eq. (19). The CLF values are those obtained using PIM and Lalor Eq. (11).

\subsection{Industrial Case VEB 5 Subsystems Model}

The CLF values between directly coupled subsystems are plotted on Fig. (19). Those values are compared to the values of the CLF obtained using the analytical formulation of Eq. (27) for perpendicular plates coupled subsystems, that have the same geometrical (surface) and material (mass, Young Modulus) properties than the Finite Element Model subsystems. So, the behavior of the model, composed of truncated cones is assumed to be close to the behavior of plates. It is a reasonable hypothesis at very high frequencies, beyond the ring frequencies. Consequently, at high 

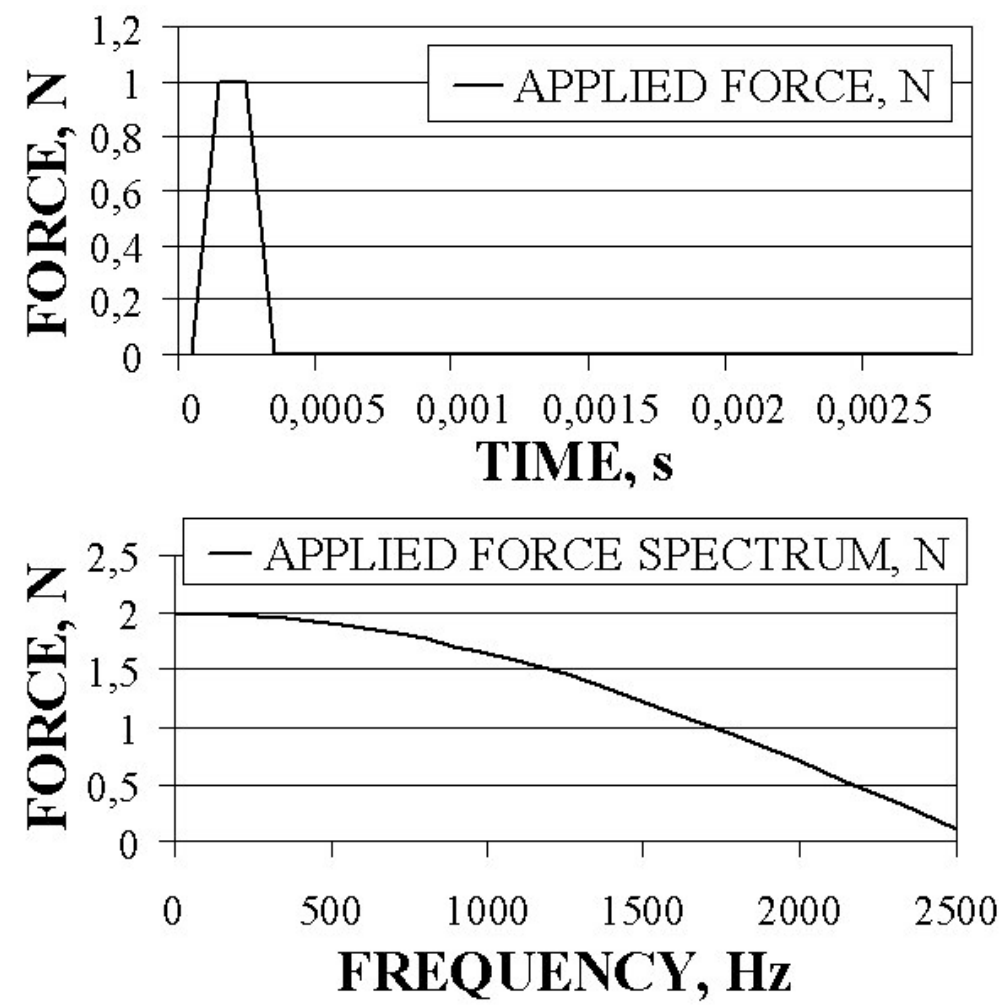

Fig. (18). Definition of point loads in the time domain (on top) and in the frequency domain (on bottom).

frequencies, computed CLF values should converge to closed formulation values.

Looking at Fig. (19), the expected convergence between closed formulation and numerical curves at high frequencies does not occur in the case of coupling between subsystems 1 and 2. This is due to the fact that the coupling between a conical and a cylindrical shell is different from the coupling between two perpendicularly coupled plates. But, a tendency is observed.

\subsection{Industrial Case VEB 3 Subsystems Model}

CLF values between each directly coupled subsystem are plotted on Figs. $(\mathbf{2 0}, \mathbf{2 1})$. The convergence of the CLF values obtained by analytical formulation and by numerical PIM can be observed at high frequencies. At mid and lower frequencies, the CLF obtained using the numerical PIM can be considered as more realistic than the analytical CLF because the Finite Element Model can take into account the real geometry and junctions. The use of SEA-Like is, of course, especially recommended to obtain accurate results in those frequency regimes.

\section{CONCLUSIONS}

This study has demonstrated the feasibility of an approach based on FE computations using explicit codes, combined with energy-based modeling, SEA-Like method and numerical method, to improve the estimate of structureborne transmissions in mid and high frequency range. It has been shown that the use of OLH method and influence circle

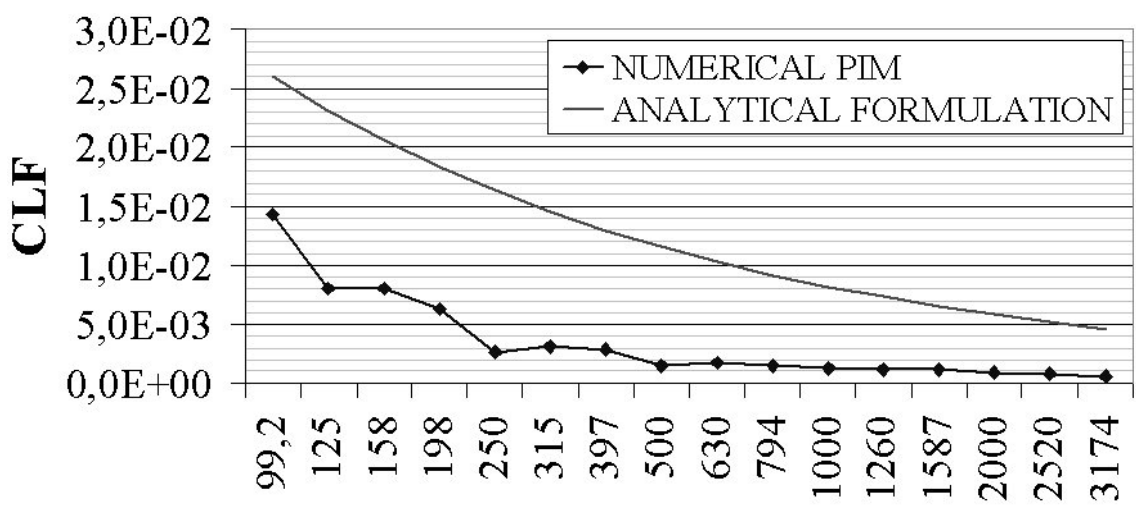

\section{THIRD OCTAVE FREQUENCY BANDS, Hz}

Fig. (19). Industrial Case: ARIANE 5 VEB. Comparison of CLF between subsystems 1 and 2 (directly connected) provided by numerical PIM and by closed formulation. 


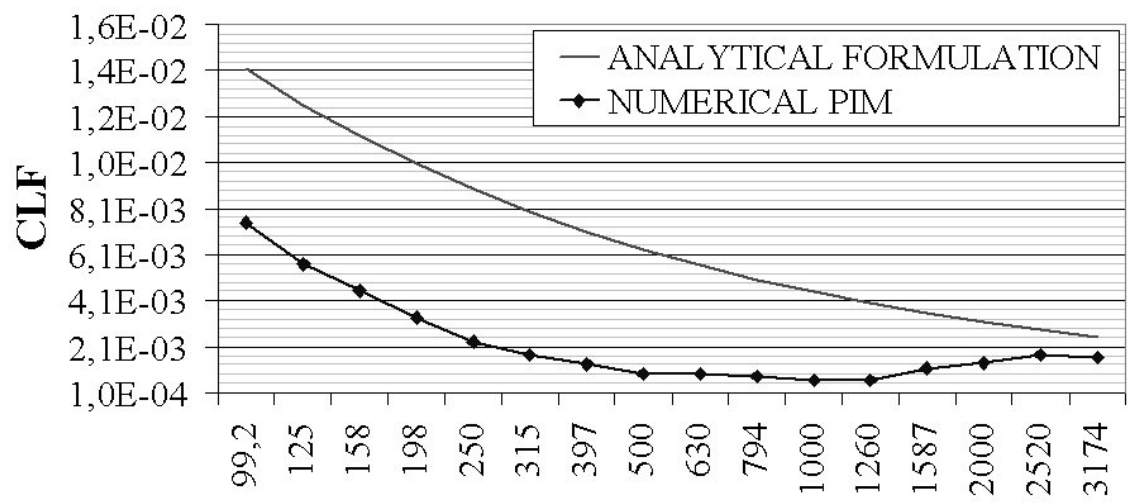

THIRD OCTAVE FREQUENCY BANDS, Hz

Fig. (20). Industrial Case: Reduced ARIANE 5 VEB (three subsystems). Comparison of CLF between subsystems 3 and 4 (directly connected) provided by numerical PIM and by closed formulation.

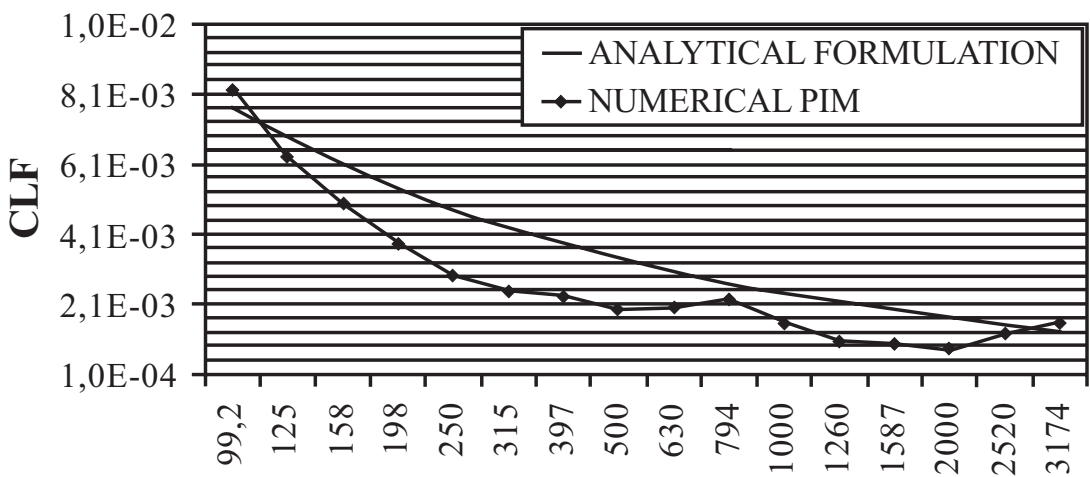

THIRD OCTAVE FREQUENCY BANDS, Hz

Fig. (21). Industrial Case: Reduced ARIANE 5 VEB (three subsystems). Comparison of CLF between subsystems 4 and 5 (directly connected) provided by numerical PIM and by closed formulation.

criterion allow the estimate of CLF and EIC with accuracy. This methodology has successfully been applied to an academic (two and three coupled plates) and to industrial cases (ARIANE $5 \mathrm{VEB}$ ). The complete process required for computing CLF and EIC has been industrialized. Computations times are reasonable (about five hours per computation of CLF and IEC). Further developments based on transient energy formulations [21-23] will be considered in order to address the mid-frequency range for further types of excitations in the mid-frequency range.

Table 1. Rigidity of the Subsystems

\begin{tabular}{|c|c|}
\hline Subsystem & Flexure Rigidity $\left(\right.$ GPa.mm (3) $^{3}$ \\
\hline $1-$ - DUMMY CU CASA » & 424,94 \\
\hline $2-\ll$ laminate CASA » & 295,42 \\
\hline $3-\ll$ EPS CONE skins » & 12,00 \\
\hline $4-\ll$ VEB skins » & 23,41 \\
\hline $5-\ll$ upper cylinder skin » & 13,38 \\
\hline
\end{tabular}

\section{ACKNOWLEDGMENTS}

Thanks to EADS ASTRIUM Space Transportation and ALTAIR Development France for their support.

\section{NOMENCLATURE}

A

$A_{i j} \quad=$ Term of Energy Influence Coefficient (EIC) matrix, raw $i$ and column $j$ (s)

CLF $=$ Coupling Loss Factor

$D \quad=$ Plate flexural rigidity $\left(\mathrm{Nm}^{2}\right)$

DLF $=$ Dissipation Loss Factor

$E i \quad=$ Total energy of subsystem $i(\mathrm{~J})$

EIC $=$ Energetic Influence Coefficient EIC

$\langle E\rangle_{s} \quad=\quad$ Averaged total energy space $(\mathrm{J})$

$\left\langle E_{i j}\right\rangle \quad=$ Term of space and frequency averaged energy matrix, raw $i$ and column $j(\mathrm{~J})$

EIC $=$ Energy Influence Coefficients

$F \quad=$ Applied force $(\mathrm{N})$

FEM $=$ Finite Element Method

$k_{b} \quad=$ Flexural wave number $\left(\mathrm{m}^{-1}\right)$

LH $=$ Latin Hypercube 


$$
\begin{aligned}
& L_{i j}=\text { Length of coupling line between subsystems } \\
& i \text { and } j(\mathrm{~m}) \\
& l_{\text {mesh }}=\text { Finite Element Model mesh average } \\
& \text { distance between } 2 \text { nodes of one element }(\mathrm{m}) \\
& M \quad=\text { Total mass of target subsystem }(\mathrm{kg}) \\
& m_{k} \quad=\text { Mass associate } \mathrm{d} \text { with node } k(\mathrm{~kg}) \\
& N=\text { Number of nodes of target subsystem, or } \\
& \text { number of samples } \\
& n_{i} \quad=\text { Modal density of subsystem } i \text { (number of } \\
& \text { modes in specified frequency band) } \\
& \text { nbloads }_{\text {optim }}=\text { Optimal number of loads } \\
& \mathrm{OLH}=\text { Optimised Latin Hypercube } \\
& P_{i, i n j} \quad=\text { Power input of subsystem } i(\mathrm{~W}) \\
& p \quad=\text { Number of sampling variables } \\
& \text { PIM } \quad=\text { Power Injection Method } \\
& \operatorname{Re} \quad=\text { Real part of a complex number } \\
& \text { SEA } \quad=\text { Statistical Energy Analysis } \\
& S_{\text {plate }} \quad=\text { Surface of the plate }\left(\mathrm{m}^{2}\right) \\
& t_{i} \quad=\text { Peak time of the } i \text { th impact (s) } \\
& u_{k} \quad=\text { Velocity of node } k\left(\mathrm{~m} / \mathrm{s}^{2}\right) \\
& \left\langle v^{2}\right\rangle=\text { Space averaged nodal velocity }\left(\mathrm{m} / \mathrm{s}^{2}\right) \\
& \bar{V} \quad=\text { Complex conjugate of averaged nodal } \\
& \text { velocity }\left(\mathrm{m} / \mathrm{s}^{2}\right) \\
& \delta \quad=\text { Dirac function } \\
& \eta_{i i} \quad=\text { Dissipation Loss Factor (DLF) of subsystem } i \\
& \eta_{i j} \quad=\text { Coupling Loss Factor (CLF) between } \\
& \text { subsystems } i \text { and } j, i \neq j \\
& \eta_{\text {itot }}=\text { Total coupling loss factor of subsystem } i \\
& \lambda=\text { Flexural wavelength }(\mathrm{m}) \\
& \rho_{\mathrm{inf}} \quad=\text { Influence circle radius }(\mathrm{m}) \\
& \rho_{s} \quad=\text { Plate surfacic mass }(\mathrm{kg}) \\
& \tau_{i j} \quad=\text { Averaged transmission coefficient between } \\
& \text { subsystems } i \text { and } j \\
& =\text { Angular frequency }(\mathrm{rad} / \mathrm{s})
\end{aligned}
$$

\section{REFERENCES}

[1] Troclet B, Mathiot N, Rivière P, Schott M. O, Ravier C. Low and High Frequency Prediction of the Vibroacoustic Response of Launch Vehicles and Aircrafts. In: Balkema AA, Eds. New Advances in Modal Synthesis of Large Structures, Brookfield: Rotterdam 1997; pp. 279-91.

[2] Lyon RH. Statistical Energy Analysis of Dynamical Systems. MIT Press: Cambridge, Massachusetts 1975.

[3] Lyon RH, Dejong RG. Theory and application of Statistical Energy Analysis, $2^{\text {nd }}$ ed. Butterworth-Heinemann: Boston 1995.

[4] Troclet B. High Frequency Prediction Using SEA in Space Industry: Proceedings of the 4th Conference of Noise Control; January 2001; University of Patras, Greece 2003.

[5] Mace B. Statistical energy analysis: coupling loss factors, indirect coupling and system modes. J Sound Vib 2005; 279: 141-70.

[6] Lyon RH, Eichler E. Random Vibration of Connected Structures. J Acoust Soc Am 1964; 36(7): 1344-54.

[7] Bies DA, Hamid S. In situ determination of loss and coupling factors by the Power Injection Method. J Sound Vib 1980; 70(2): 187-204.

[8] Plunt J. Power Injection Method for vibration damping determination of body panels with applied damping treatments and trim. SAE Transac 1991; 100 (6): 1563-71.

[9] De Langhe K. High frequency vibrations: contributions to experimental and computational SEA parameter identification techniques. PhD Thesis, KUL, Leuven 1996.

[10] Fredö CR. Statistical energy analysis and the individual case. PhD Thesis, Chalmers University of Technology. Gothenburg 1995.

[11] Lalor $\mathrm{N}$. The experimental determination of vibrational energy balance in complex structure. Conference on stress and vibration. Paper $n^{\circ} 108429$. Londres 1989.

[12] Guyader JL, Boisson C, Lesueur C. Energy transmission in finite coupled plate: part 1: theory. J Sound Vib 1982; 81(1): 81-92.

[13] Guyader JL, Boisson C, Lesueur C. Rayonnement acoustique des structures. Collection EDF-GDF. Eyrolles: Paris 1988.

[14] Hiverniau B, Troclet B, Ichchou MN, Jézéquel L. Development of Hybrid FEM/SEA Methods for Vibroacoustic Mid and High Frequency Predictions for Aeroacoustic Excitations: Proceedings of 9th international conference on recent advances in structural dynamics. July 2006; Southampton.

[15] Ichchou MN, Hiverniau B, Troclet B. Equivalent 'rain on the roof' loads for random spatially correlated excitations in the mid-high frequency range. J Sound Vib doi:10.1016/j.jsv.2008.11.050.

[16] Harris CM, Crede CE. Shock and Vibration Handbook, $2^{\text {nd }}$ ed. Mc Graw Hill: New York 1976

[17] Kayvantash K, Delcroix F, Bekkour T, Marro M. DSS (HyperstudyDSS - Altair Development France) Theory User Guide. 2005

[18] Liefvendahl M. A study on algorithms for optimization of Latin hypercubes. J Stat Plan Interference 2006; 136: 3231-47.

[19] Pope LD, Wilby JF. Band-limited power flow into enclosures. J Acoust Soc Am 1977; 62(4): 906-11.

[20] Pope LD, Wilby JF. Band-limited power flow into enclosures II. J Acoust Soc Am 1977; 67(3): 823-26.

[21] Ichchou MN, Lebot A, Jezequel L. A transient local energy approach as an alternative to transient SEA: wave and telegraph equations. J Sound Vib 1982; 81(1): 81-92.

[22] Ichchou MN, Akrout S, Mencik JM. Guided waves group and energy velocities via finite elements. J Sound Vib 2007; 305(4-5): 931-44.

[23] Sui F, Ichchou MN, Jezequel L. Prediction of vibroacoustics energy using a discretized transient local energy approach and comparison with TSEA. J Sound Vib 2002; 251(1): 160-80. 